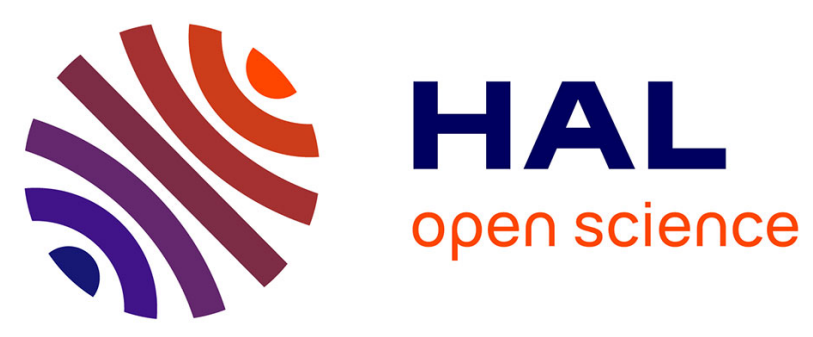

\title{
Room-Temperature Synthesis of Tetrasubstituted 1,3-Dithioles by Dimerizing Sulfuration of Chalcones with Elemental Sulfur
}

\author{
T .B. Nguyen, Dinh Hung Mac, Pascal Retailleau
}

\section{- To cite this version:}

T .B. Nguyen, Dinh Hung Mac, Pascal Retailleau. Room-Temperature Synthesis of Tetrasubstituted 1,3-Dithioles by Dimerizing Sulfuration of Chalcones with Elemental Sulfur. Journal of Organic Chemistry, 2020, 85 (21), pp.13508-13516. 10.1021/acs.joc.0c01618 . hal-02993853

\section{HAL Id: hal-02993853 \\ https://hal.science/hal-02993853}

Submitted on 24 Nov 2020

HAL is a multi-disciplinary open access archive for the deposit and dissemination of scientific research documents, whether they are published or not. The documents may come from teaching and research institutions in France or abroad, or from public or private research centers.
L'archive ouverte pluridisciplinaire HAL, est destinée au dépôt et à la diffusion de documents scientifiques de niveau recherche, publiés ou non, émanant des établissements d'enseignement et de recherche français ou étrangers, des laboratoires publics ou privés. 


\title{
Room Temperature Synthesis of Tetrasubstituted 1,3-Dithioles by
}

\section{Dimerizing Sulfuration of Chalcones with Elemental Sulfur}

\author{
Thanh Binh Nguyen, ${ }^{\mathrm{a}, *}$ Dinh Hung Mac, ${ }^{\mathrm{b}, *}$ and Pascal Retailleau ${ }^{\mathrm{a}}$ \\ a Institut de Chimie des Substances Naturelles, CNRS UPR 2301, Université Paris-Sud, Université \\ Paris-Saclay, 1 avenue de la Terrasse, 91198 Gif-sur-Yvette, France \\ ${ }^{b}$ Faculty of Chemistry, VNU University of Science, Vietnam National University in Hanoi, 19 Le \\ Thanh Tong, Hanoi, Viet Nam \\ Email: macdinhhung@hus.edu.vn \\ thanh-binh.nguyen@enrs.fr
}

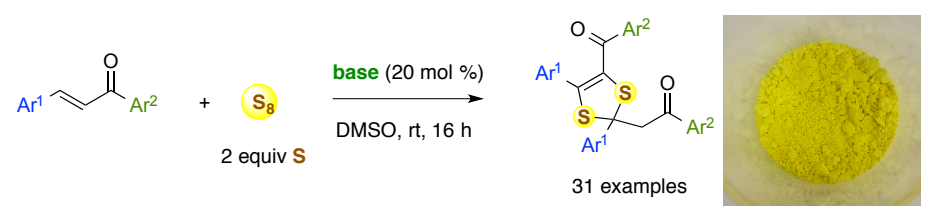

\begin{abstract}
Chalcones were found to undergo a sulfurative dimerization with elemental sulfur to tetrasubstituted 1,3-dithioles. The reaction was found to proceed at room temperature in the presence of a nitrogen base catalyst in DMSO.
\end{abstract}

\section{Introduction}

Organosulfur compounds, including sulfa heterocycles, are ubiquitous in both natural products and artificial molecules with interesting properties. Consequently, the finding of efficient and direct approaches to such molecules starting from simple starting materials and readily available in terms of both quantity and variety plays undoubtedly a central role in accelerating their research and development.

To this end, a wide range of synthetic methods has been developed to incorporate one or more sulfur atoms to the organic scaffolds using simple inorganic sulfur sources. Among these sulfur precursors, elemental sulfur is unarguably the best sulfur source. Using elemental sulfur in organic synthesis, we can benefit from its unique redox chemistry as an element, its user-friendliness along with its easy 
availability. Consequently, this element has drawn the attention of chemists to develop cost-effective and environmentally friendly reactions in recent years. ${ }^{1}$

Among sulfa heterocycles, 1,3-dithioles are well known for a wide range of applications with some representative examples we shown in Figure 1.

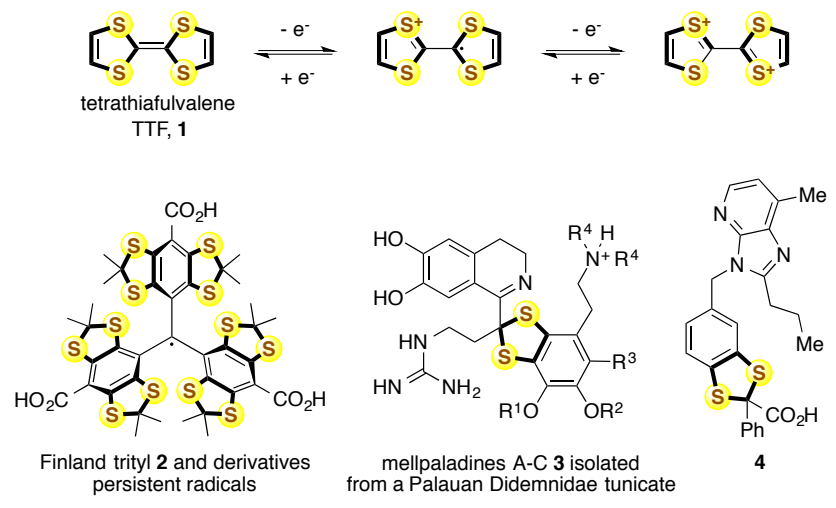

Figure 1. Selected examples of 1,3-dithioles

Tetrathiafulvalene (TTF) unit and derivatives are well known electron donors with three stable redox states. Thanks to their capability of forming easily charge-transfer complexes with electron acceptors, TTF is the core structure for development of organic conducting materials with over 13,000 scientific publications. ${ }^{2}$ Interestingly, stabilized by fused 1,3-dithiole rings, tetrathiatriarylmethyl (TAM) radicals derived from the Finland trityl $\mathbf{2}$ are considerably more persistent than the Gomberg radical even under in-cell conditions. With strong EPR signals, TAM derivatives could be used for magnetic resonance imaging, dynamic nuclear polarization, and spin labeling EPR. ${ }^{3}$ Recently, tunicate guanidine alkaloids mellpaladines A-C 3, were found to bind to synaptic receptors, and modulated behavioral profiles of mice. ${ }^{4}$ Furthermore, 1,3-benzodithiole-2-carboxylates 4 displayed potent binding affinity to the angiotensin II AT1 receptor. $^{5}$

Consequently, a collection of synthetic methods of this scaffold was developed. Most of them were based on reactions of 1,2-dithiole-3-thiones or thioesters with activated acetylenes, ${ }^{6}$ reactions involving sulfur-rich compounds ${ }^{7}$ as well as elemental sulfur ${ }^{8}$ as the only sulfur source. In the latter cases, most of the reactions required prolonged heating or highly reactive substrates and were applied to a very limited number of examples. If densely substituted 1,3-dithiole are of interest, synthesis of the respective precursors might be a complex and challenging multistep process. 
An interesting aspect of elemental sulfur is its capability to promote oligomerization of single starting materials to provide oligomers that are much more difficult or unpractical to obtain otherwise. Via this strategy, simple and readily available starting molecules could undergo dimerization, ${ }^{9}$ trimerization, ${ }^{10}$ tetramerization ${ }^{11}$ and hexamerization ${ }^{12}$ with various degree of sulfuration. ${ }^{13}$ Herein, we report a direct dimerization of chalcones with sulfur to provide original tetrasubstituted 1,3-dithiole scaffold. More strikingly, the reactions could be performed under simple conditions even at room temperature. ${ }^{14}$

\section{Results and Discussion}

During the course of our study on new reactivities of the combination $\mathrm{S}_{8} / \mathrm{DMSO}^{15}$ which could act as both oxidizing and sulfurating agent, we developed a three-component access to 2-aminothiophene 7 from phenylacetonitrile 5, chalcone A0 and elemental sulfur (Scheme 1). ${ }^{16}$ Reasonable yield was obtained if the Michael adduct $\mathbf{6}$ between phenylacetonitrile $\mathbf{5}$ and chalcone A0 were formed prior to the DABCO-catalyzed thiophenation with elemental sulfur. On the contrary, if three components of the reaction were mixed together in the presence of DABCO catalyst, 2-aminothiophenes 7 were formed in low yield along with pyrrole $\mathbf{8}$ from trimerization of $\mathbf{5}^{10 \mathrm{a}}$ and a byproduct $\mathbf{B 0}$ which could be generated in another control experiment performed without 5 (Table 1, entry 1). Interestingly, high yield of B0 could be achieved even at rt. The exact structure of B0 was unambiguously determined to be a tetrasubstituted dithiole by single crystal X-ray crystallographic analysis.

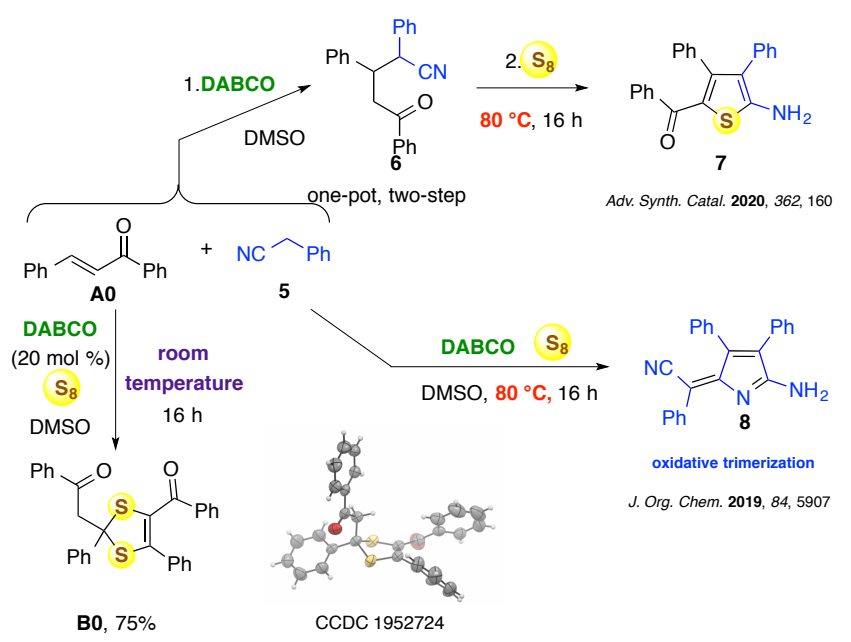

Scheme 1. Initial observation for the formation of dichalcone disulfide Bo 
Other basic additives such as liquid organic bases (triethylamine, tri- $n$-propylamine, $t$-butylamine, DIPEA, $N$-methylpiperidine, 2,2,6,6-tetramethylpiperidine, $N$-methylpyrrolidine in stoichiometric amount) (Table 1, entries 2-10) or solid inorganic base ( $\mathrm{CsF}, \mathrm{Na}_{2} \mathrm{~S} \cdot 5 \mathrm{H}_{2} \mathrm{O}, \mathrm{K}_{2} \mathrm{CO}_{3}$ in 20 mol \% amount) were also found to promote this sulfurative dimerization in good yields in almost all cases (Table 1, entries 11-13).

\section{Table 1. Screening of the reaction conditions}

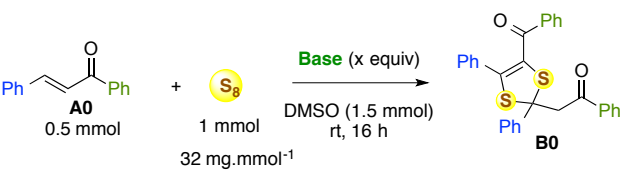

\begin{tabular}{|c|c|c|}
\hline Entry $^{a}$ & Base (x equiv) & Yield $(\%)^{b}$ \\
\hline 1 & DABCO (0.2) & 75 \\
\hline 2 & $\mathrm{NEt}_{3}(1)$ & 75 \\
\hline 3 & $\mathrm{~N}(n-\operatorname{Pr})_{3}(1)$ & 70 \\
\hline 4 & $t-\mathrm{BuNH}_{2}(1)$ & 60 \\
\hline 5 & DIPEA (1) & 71 \\
\hline 7 & $N$-methylpiperidine (1) & 67 \\
\hline 8 & 2,2,6,6-tetramethylpiperidine (1) & 52 \\
\hline 9 & $N$-methylpyrrolidine (1) & 55 \\
\hline 10 & DBU (1) & 73 \\
\hline 11 & $\operatorname{CsF}(0.2)$ & 70 \\
\hline 12 & $\mathrm{Na}_{2} \mathrm{~S} \cdot 5 \mathrm{H}_{2} \mathrm{O}(0.2)$ & 62 \\
\hline 13 & $\mathrm{~K}_{2} \mathrm{CO}_{3}(0.2)$ & 55 \\
\hline 14 & $\mathrm{NEt}_{3}(\mathbf{0 . 2 )}$ & 75 \\
\hline 15 & $\mathrm{NEt}_{3}(0.1)$ & 47 \\
\hline
\end{tabular}




$\begin{array}{llc}16^{c} & \mathrm{NEt}_{3}(0.2-10) & 0 \\ 17^{d} & \mathrm{NEt}_{3}(0.2) & 50 \\ 18^{e} & \mathrm{NEt}_{3}(0.2) & 30\end{array}$

${ }^{a}$ Reaction conditions: chalcone A0 (0.5 mmol, $\left.104 \mathrm{mg}\right), \mathrm{S}(1 \mathrm{mmol} 32 \mathrm{mg})$, base (x equiv), DMSO (1.5 mmol, $0.1 \mathrm{~mL})$ at $\mathrm{rt} .{ }^{b}$ Isolated yield. ${ }^{c}$ Reaction performed in the absence of DMSO. ${ }^{d}$ Reaction performed in DMF $(0.1 \mathrm{~mL})$ instead of DMSO. ${ }^{e}$ Reaction performed with $\mathrm{S}$ (1 equiv, $0.5 \mathrm{mmol}, 16 \mathrm{mg}$ ). Comparably good yield $75 \%$ was obtained when triethylamine was used in catalytic amount (20 mol \%) (entry 14$)$. Lowering $\mathrm{NEt}_{3}$ amount to $10 \mathrm{~mol} \%$ resulted in lower yield (54\%, entry 15$)$. When reaction was performed in the absence of DMSO, chalcone $\mathbf{A 0}$ remained unchanged regardless of $\mathrm{NEt}_{3}$ amount (entry 16). We noted that in this case, since both chalcone A0 and sulfur are solid at $\mathrm{rt}$, their mixtures with $\mathrm{NEt}_{3}$ remained slurry states with increasing amounts of $\mathrm{NEt}_{3}(0.2-10$ equiv). When DMF was used instead of DMSO (entry 17), the reaction was not complete, the expected dimer B0 was formed in low yield along with many side-products.

When only 1 equiv sulfur was used in the reaction, i.e. to provide exactly two sulfur atoms in dimer product B0, a sharp drop lower yield occurred along with the formation of unidentified products (entry 18). Since the expected sulfurative dimerization reaction required the removal of one hydrogen atom per each consumed chalcone, it is clear that sulfur acted also as a dehydrogenative agent.

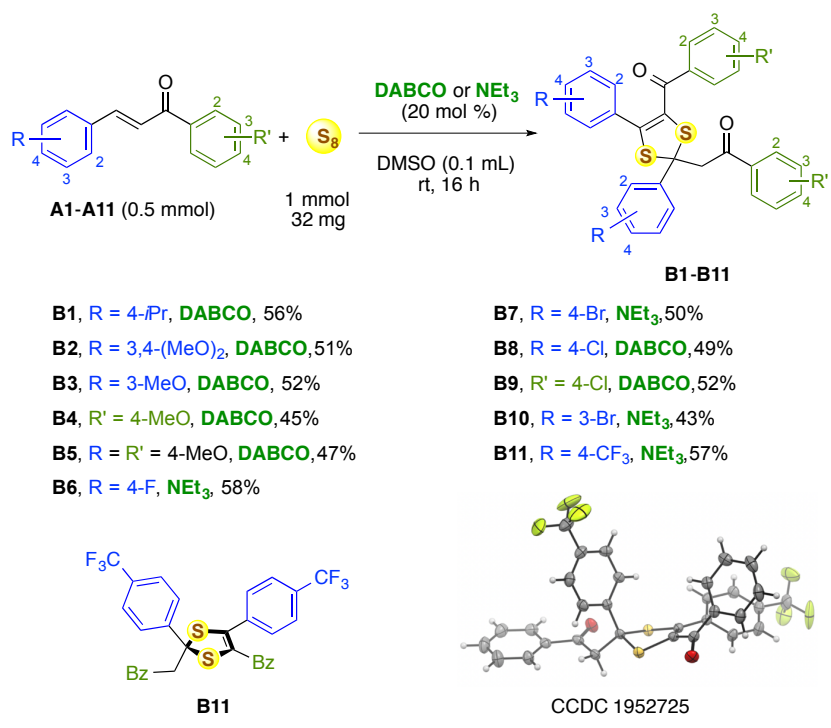

Scheme 2. 
Gratifyingly, the dimer B0 could be readily precipitated as a bright yellow solid by simply diluting the reaction mixtures with methanol and thus readily isolated by filtration.To further demonstrate the efficiency of this procedure, we explored the reaction with chalcones A1-A11 (Scheme 2).

Two sets of optimized conditions involving either DABCO (entry 1) or $\mathrm{NEt}_{3}$ (entry 14) in $20 \mathrm{~mol}_{\%}$ amount were employed. A wide range of functional substituents (alkyl A1, alkoxy A2-A5, halogen A6A10, trifluoromethyl A11) in either or both aromatic rings were shown to be compatible with the reaction conditions. The structure of disulfide dimers B11 was determined by X-ray diffraction, confirming again the common 1,3-dithiole scaffold of all products obtained by our reaction.

Next, a series of naphthalene and heterocyclic chalcones A12-A30 was tested and these substrates gave moderate yields ranging from $40 \%$ to $59 \%$ (Scheme 3). Chalcones bearing naphthyl substituents A12A21 as well as heterocyclic ring such as thienyls (A21-A24), benzothienyl (A25), pyridyls (A26-A29) and furyl (A30) displayed good reactivity, providing the corresponding disulfurative dimeric products

\section{B12-B30.}

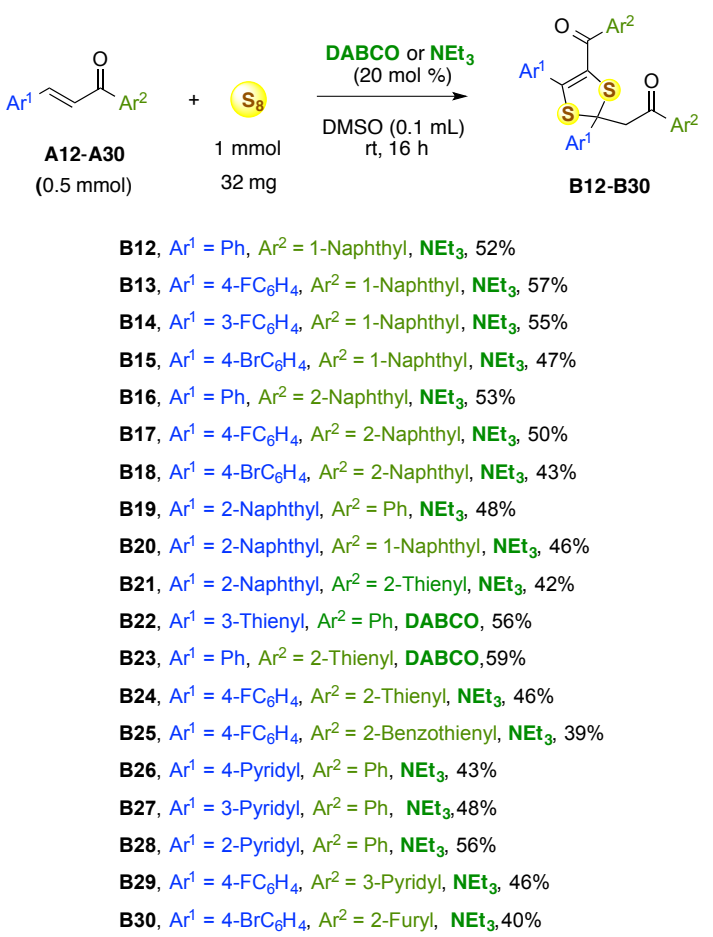

Scheme 3. 
We emphasized that the best yield was obtained with the most simple chalcone A0, which could be readily crystalized out from its reactions mixture by simply diluting with methanol, whereas other dimers B1-B30 were shown to be much more soluble than in this solvent and thus could not be efficiently isolated by this manner. It is possible that the higher crystallinity of $\mathbf{B 0}$ allowed this dimer to be separated from the reaction mixture when it was formed and thus avoided other side reactions that could lower the global yields.

To gain further insight into the reaction mechanism, several control experiments were performed. First, $\mathrm{NEt}_{3}$ ( $\mathrm{p} K_{\mathrm{b}}$ 3.25) was replaced by a weaker base in stoichiometric amount (1 equiv) such as $\mathrm{N}$ methylmorpholine ( $K_{\mathrm{b}}$ 6.62) and pyridine ( $K_{\mathrm{b}}$ 8.75) (Scheme 4). Chalcone A0 was converted only partially to dimer B0 (30\% conversion) with $N$-methylmorpholine (1 equiv) at $\mathrm{rt}$ and remained unchanged with the lower loading of this base $(20 \mathrm{~mol} \%)$ or with pyridine (1 equiv) even upon heating up to $80^{\circ} \mathrm{C}$, indicating that the formation of sulfur-base adduct via ring opening of $\mathrm{S}_{8}$ by the base was a key step in initiating the reaction. This step depended on both basicity and concentration of the base.

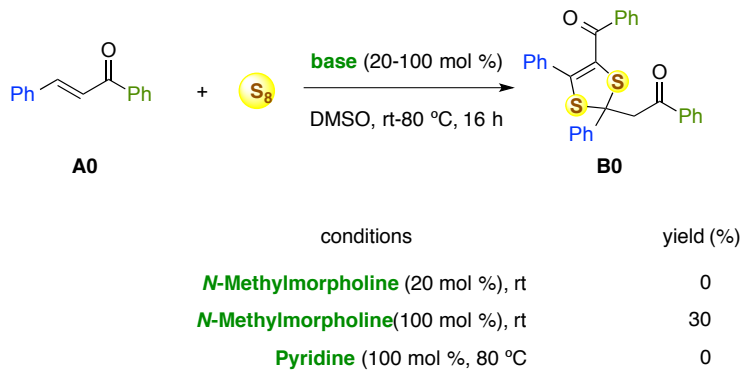

\section{Scheme 4. Control experiment with weaker bases additives}

Second, we found that chalcone substrates bearing a 2-substituent such as 2-fluoro-, 2-chloro-, 2-bromochalcones A31-A33 or 1-naphthyl analog A34 did not undergo the expected sulfurative dimerization (Scheme 5). Their reactions under the same conditions led to complex mixtures in which we could detect easily the presence of dihydrochalcone issued from reduction of the olefin based on their ${ }^{1} \mathrm{H}$ NMR signals of aliphatic protons. This side reaction was previously observed with $o$-halochalcones. ${ }^{17 a}$ From our previous studies on sulfur-chalcone reactions, ${ }^{15 f, 17}$ we speculated that the reaction pathway should be initiated by a Michael nucleophilic addition reaction to the chalcone and an ortho substituent on the ring $\mathrm{B}$ of chalcone substrate would slower or even inhibit the reaction. 


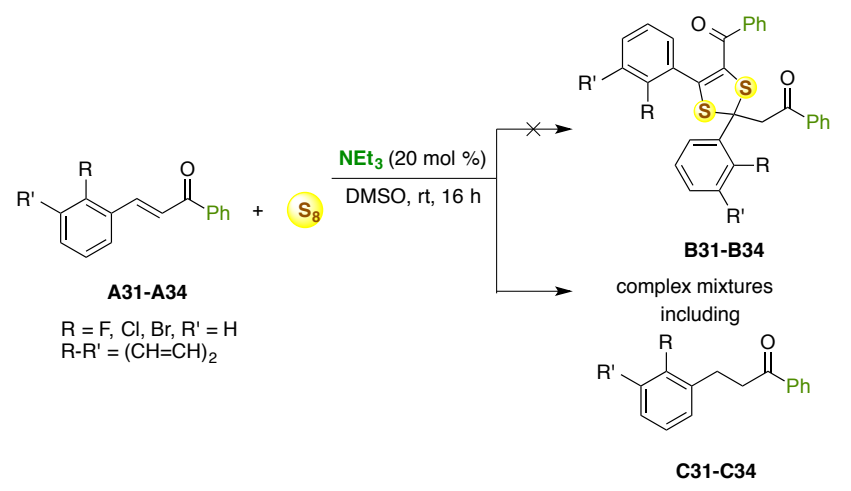

Scheme 5. Failed reactions with $o$-substituted chalcones

Based on the obtained results, the above-mentioned control experiments, and the precedent literature, ${ }^{18}$ a plausible reaction mechanism is outlined in Scheme 6.
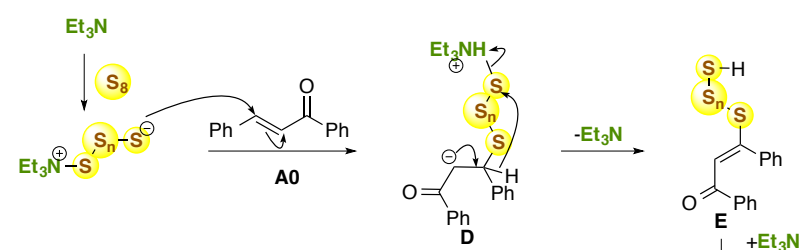

AO $\begin{gathered}+\mathrm{Et}_{3} \mathrm{~N} \\ -\mathrm{Et}_{3} \mathrm{NH}^{+}\end{gathered}$

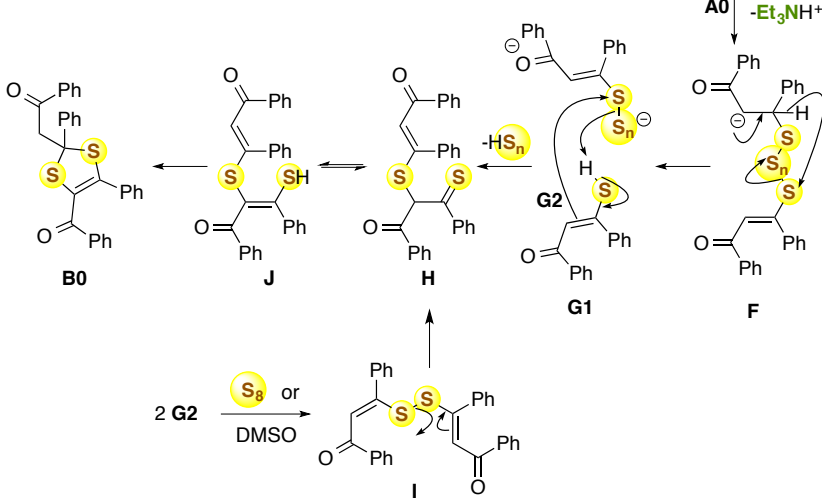

\section{Scheme 6. Plausible reaction mechanism}

The reaction was initiated by a Michael addition of $\mathrm{NEt}_{3}$-sulfur adduct to chalcone $\mathbf{A 0}$. The resulting adduct $\mathbf{D}$ underwent a disproportionation in which the sulfurated chalcone moiety was oxidized and the terminal N-S bond was reduced, leading to polysulfane $\mathbf{E}$. Subsequent addition of $\mathbf{E}$ to another molecule of chalcone A1, followed by fragmentation of the resulting adduct $\mathbf{F}$ would give two fragments $\mathbf{G 1}$ and G2, different only in the number of sulfur atom and interconvertible. The transformation of $\mathbf{G} \mathbf{2}$ into thioketone $\mathbf{H}$ could proceed by combining with $\mathbf{G 1}$ with the simultaneous extrusion of hydropolysulfide 
or by $\mathrm{S}_{8-}$ or DMSO- promoted oxidative dimerization into $\mathbf{I}$ then rearrangement. ${ }^{18}$ Cyclization of enethiol $\mathbf{J}$, which is a tautomer of $\mathbf{H}$, would finally provide the dithiole $\mathbf{B 0}$.

In conclusion, we explored the reactivity of elemental sulfur on chalcones and developed an efficient strategy for the construction of tetrasubstituted 1,3-dithiole compounds using $\mathrm{NEt}_{3}$ in DMSO as a promoter. The reaction went through a sulfurative dimerization of chalcone under mild conditions with broad substrate scope. Densely substituted 1,3-dithioles were obtained in reasonable yields for the majority of tested substrates. The result presented here confirms once again the very rich organic chemistry of elemental sulfur even under very simple conditions. Since chalcones are readily available in a wide range of structures from both commercial suppliers and syntheses, our method opens a new and convenient access to a large collection of original structures for bioactive screening. Further experiments on the mechanism as well as synthetic application are currently ongoing in our laboratory.

\section{Experimental Section}

\section{General Information}

Reagents were obtained from commercial supplier and used without further purification. Analytical thin layer chromatography (TLC) was purchased from Merck KGaA (silica gel 60 F254). Visualization of the chromatogram was performed by UV light $(254 \mathrm{~nm})$ or $\mathrm{KMnO}_{4}$ or vanilline stains. Flash column chromatography was carried out using kieselgel 35-70 $\mu \mathrm{m}$ particle sized silica gel (230-400 mesh). NMR Chemical shifts are reported in ( $\delta$ ) ppm relative to tetramethylsilane (TMS) with the residual solvent as internal reference $\left(\mathrm{CDCl}_{3}, \delta 7.26 \mathrm{ppm}\right.$ for ${ }^{1} \mathrm{H}$ and $\delta 77.0 \mathrm{ppm}$ for $\left.{ }^{13} \mathrm{C}\right)$. Data are reported as follows: chemical shift, multiplicity $(\mathrm{s}=$ singlet, $\mathrm{d}=$ doublet, $\mathrm{t}=$ triplet, $\mathrm{q}=$ quartet, $\mathrm{m}=$ multiplet), coupling constants $(\mathrm{Hz})$ and integration. Mass spectra were obtained using electron spray ionization (ESI). The HRMS data were measured on MALDI-TOF instrument.

\section{General procedure}

A mixture of chalcone A ( $0.5 \mathrm{mmol}, 1$ equiv), $\mathrm{S}(32 \mathrm{mg}, 1 \mathrm{mmol}, 2$ equiv), triethylamine or DABCO $(0.1 \mathrm{mmol})$ in $0.1 \mathrm{~mL}$ of DMSO in a 7-mL test-tube closed with a septum was stirred at $\mathrm{rt}$ for $16 \mathrm{~h}$. In case of A0, upon dilution with $\mathrm{MeOH}(2-4 \mathrm{~mL})$, dimer $\mathrm{B} 0$ was precipitated as a bright yellow solid, which was filtered, washed with $\mathrm{MeOH}(2 \mathrm{~mL}$ x 3$)$ and dried in vacuo to give pure B0. In cases of chalcones A1-A30, due to higher solubilities of the corresponding dimers B1-B30, the crude mixture 
was diluted with $\mathrm{MeOH}: \mathrm{CH}_{2} \mathrm{Cl}_{2}(4 \mathrm{~mL}$, v:v 1:1) and filtered over Celite. After removal of volatiles in vacuo, the residue was purified by column chromatography (hexane/ethyl acetate: 19/1 to 9/1) to give the dimer product a bright yellow solid.

Large-scale synthesis of B0: A mixture of chalcone A0 (5.20 g, $25 \mathrm{mmol}), \mathrm{S}$ (1.60 g, $50 \mathrm{mmol})$, and DABCO $(560 \mathrm{mg}, 5 \mathrm{mmol})$ in $5.0 \mathrm{~mL}$ of DMSO in a $25-\mathrm{mL}$ round-bottom flask closed with a septum was stirred at $\mathrm{rt}$ for $16 \mathrm{~h}$. Dilution of the reaction mixture with $\mathrm{MeOH}(20 \mathrm{~mL})$ precipitated the yellow product, which was filtered and washed with $\mathrm{MeOH}(2 \mathrm{~mL} \times 5)$. The yellow powder was dried in vacuo $(4.18 \mathrm{~g}, 70 \%)$.

\section{Characterization of the products}

\section{2-(4-Benzoyl-2,5-diphenyl-1,3-dithiol-2-yl)-1-phenylethan-1-one (B0)}<smiles>O=C(CC1(c2ccccc2)SC(C(=O)c2ccccc2)=C(c2ccccc2)S1)c1ccccc1</smiles>

Upon dilution with $\mathrm{MeOH}(2-4 \mathrm{~mL})$, the product was precipitated as a bright yellow solid, which was filtered, washed with $\mathrm{MeOH}(2 \mathrm{~mL}$ x 3$)$ and dried in vacuo to give pure $\mathbf{B 0}$.

Bright yellow solid (90 mg, 75\%).

${ }^{1} \mathrm{H}$ NMR $\left(300 \mathrm{MHz}, \mathrm{CDCl}_{3}\right) \delta 8.0(\mathrm{~d}, J=7.7 \mathrm{~Hz}, 2 \mathrm{H}), 7.9(\mathrm{~d}, J=7.9 \mathrm{~Hz}, 2 \mathrm{H}), 7.6(\mathrm{t}, J=7.4 \mathrm{~Hz}, 1 \mathrm{H})$, $7.6-7.4(\mathrm{~m}, 3 \mathrm{H}), 7.4(\mathrm{t}, J=7.6 \mathrm{~Hz}, 2 \mathrm{H}), 7.4-7.2(\mathrm{~m}, 4 \mathrm{H}), 7.2-7.0(\mathrm{~m}, 5 \mathrm{H}), 4.5(\mathrm{~s}, 2 \mathrm{H})$.

${ }^{13} \mathrm{C}\left\{{ }^{1} \mathrm{H}\right\} \mathrm{NMR}\left(75 \mathrm{MHz}, \mathrm{CDCl}_{3}\right) \delta 194.7,190.4,144.5,142.2,136.7,136.2,133.7,132.5,132.1,129.9$, $129.5,129.3,128.8,128.4,128.2,128.1,128.0,127.9,126.5,66.4\left(\mathrm{CS}_{2}\right), 51.2$.

HRMS m/z calculated for $[\mathrm{M}+\mathrm{Na}]^{+} \mathrm{C}_{30} \mathrm{H}_{22} \mathrm{NaO}_{2} \mathrm{~S}_{2}$ 501.0959. Found 601.0963.

2-(4-Benzoyl-2,5-bis(4-isopropylphenyl)-1,3-dithiol-2-yl)-1-phenylethan-1-one (B1) 


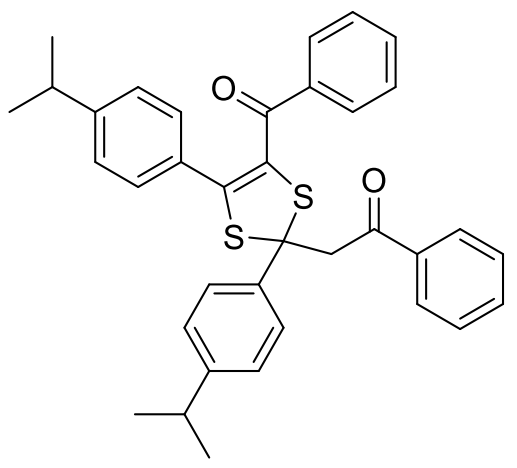

Eluent: hexane/ethyl acetate: $19 / 1$ to $9 / 1$.

Bright yellow solid (79 mg, 56\%).

${ }^{1} \mathrm{H}$ NMR $\left(300 \mathrm{MHz}, \mathrm{CDCl}_{3}\right) \delta 8.0-7.9(\mathrm{~m}, 2 \mathrm{H}), 7.8-7.7(\mathrm{~m}, 2 \mathrm{H}), 7.6-7.5(\mathrm{~m}, 1 \mathrm{H}), 7.5-7.4(\mathrm{~m}, 4 \mathrm{H})$, $7.2-7.2(\mathrm{~m}, 2 \mathrm{H}), 7.1(\mathrm{td}, J=6.2,1.7 \mathrm{~Hz}, 2 \mathrm{H}), 7.1-7.0(\mathrm{~m}, 1 \mathrm{H}), 6.9(\mathrm{~d}, J=8.2 \mathrm{~Hz}, 2 \mathrm{H}), 4.5(\mathrm{~s}, 2 \mathrm{H})$, $2.9(\mathrm{p}, J=6.9 \mathrm{~Hz}, 1 \mathrm{H}), 2.7(\mathrm{p}, J=6.9 \mathrm{~Hz}, 1 \mathrm{H}), 1.2(\mathrm{~d}, J=6.9 \mathrm{~Hz}, 6 \mathrm{H}), 1.1(\mathrm{~d}, J=6.9 \mathrm{~Hz}, 6 \mathrm{H})$.

${ }^{13} \mathrm{C}\left\{{ }^{1} \mathrm{H}\right\}$ NMR $\left(75 \mathrm{MHz}, \mathrm{CDCl}_{3}\right) \delta 194.9,190.5,150.5,148.4,145.6,139.3,136.9,133.6,132.1,130.0$, $129.2,128.7,128.1,127.8,127.7,127.7,126.4,66.0,51.2,33.8,33.6,23.8,23.6$.

HRMS m/z calculated for $[\mathrm{M}+\mathrm{Na}]^{+} \mathrm{C}_{36} \mathrm{H}_{34} \mathrm{NaO}_{2} \mathrm{~S}_{2}$ 585.1898. Found 585.1996.

\section{2-(4-Benzoyl-2,5-bis(3,4-dimethoxyphenyl)-1,3-dithiol-2-yl)-1-phenylethan-1-one (B2)}<smiles>COc1ccc(C2=C(C(=O)c3ccccc3)SC(CC(=O)c3ccccc3)(c3ccc(OC)c(OC)c3)S2)cc1OC</smiles>

Eluent: hexane/ethyl acetate: $19 / 1$ to $9 / 1$.

Bright yellow solid (76 mg, 51\%).

${ }^{1} \mathrm{H}$ NMR $\left(300 \mathrm{MHz}, \mathrm{CDCl}_{3}\right) \delta 8.0(\mathrm{~d}, J=7.3 \mathrm{~Hz}, 3 \mathrm{H}), 7.6-7.4(\mathrm{~m}, 9 \mathrm{H}), 7.3-7.2(\mathrm{~m}, 3 \mathrm{H}), 7.2-7.1$ $(\mathrm{m}, 3 \mathrm{H}), 6.9(\mathrm{dd}, J=8.3,2.1 \mathrm{~Hz}, 1 \mathrm{H}), 6.8(\mathrm{~d}, J=8.5 \mathrm{~Hz}, 1 \mathrm{H}), 6.6(\mathrm{t}, J=2.5 \mathrm{~Hz}, 1 \mathrm{H}), 6.6(\mathrm{~d}, J=8.4 \mathrm{~Hz}$, 1H), 4.5 (s, 2H), 3.9 (s, 3H), 3.9 (s, 3H), 3.8 (s, 3H), 3.7 (s, 3H). 
${ }^{13} \mathrm{C}\left\{{ }^{1} \mathrm{H}\right\} \operatorname{NMR}\left(75 \mathrm{MHz}, \mathrm{CDCl}_{3}\right) \delta 195.0,190.6,150.1,148.6,148.5,148.2,145.0,136.8,136.3,134.4$, $133.7,132.6,129.2,128.8,128.6,128.1,128.0,125.2,124.7,123.2,118.6,113.0,110.7,110.6,110.5$, $65.6,56.0,55.9,55.7,50.9$.

HRMS m/z calculated for $[\mathrm{M}+\mathrm{Na}]^{+} \mathrm{C}_{34} \mathrm{H}_{30} \mathrm{NaO}_{6} \mathrm{~S}_{2}$ 621.1381. Found 621.1387.

2-(4-Benzoyl-2,5-bis(3-methoxyphenyl)-1,3-dithiol-2-yl)-1-phenylethan-1-one (B3)<smiles>COc1cccc(C2=C(C(=O)c3ccccc3)SC(CC(=O)c3ccccc3)(c3cccc(OC)c3)S2)c1</smiles>

Eluent: hexane/ethyl acetate: $19 / 1$ to $9 / 1$.

Bright yellow solid (70 mg, 52\%).

${ }^{1} \mathrm{H}$ NMR $\left(500 \mathrm{MHz}, \mathrm{CDCl}_{3}\right) \delta 7.9(\mathrm{dd}, J=8.4,1.3 \mathrm{~Hz}, 2 \mathrm{H}), 7.6-7.5(\mathrm{~m}, 3 \mathrm{H}), 7.5-7.4(\mathrm{~m}, 3 \mathrm{H}), 7.4$ (ddd, $J=7.9,2.0,0.9 \mathrm{~Hz}, 1 \mathrm{H}), 7.3-7.3(\mathrm{~m}, 2 \mathrm{H}), 7.2-7.1(\mathrm{~m}, 2 \mathrm{H}), 7.0(\mathrm{t}, J=7.9 \mathrm{~Hz}, 1 \mathrm{H}), 6.9-6.8$ (m, 1H), $6.8(\mathrm{ddd}, J=8.2,2.5,0.9 \mathrm{~Hz}, 1 \mathrm{H}), 6.7(\mathrm{dd}, J=2.6,1.6 \mathrm{~Hz}, 1 \mathrm{H}), 6.7(\mathrm{ddd}, J=8.3,2.6,1.0 \mathrm{~Hz}$, $1 \mathrm{H})$.

${ }^{13} \mathrm{C}\left\{{ }^{1} \mathrm{H}\right\} \mathrm{NMR}\left(126 \mathrm{MHz}, \mathrm{CDCl}_{3}\right) \delta 194.8,190.4,159.6,159.1,144.1,143.9,136.8,136.3,133.8,133.3$, $132.6,129.5,129.3,129.2,128.8,128.2,128.0,126.6,122.5,118.7,115.8,115.0,113.2,113.0,66.2$, $55.4,55.3,51.3$.

HRMS m/z calculated for $[\mathrm{M}+\mathrm{Na}]^{+} \mathrm{C}_{32} \mathrm{H}_{26} \mathrm{NaO}_{4} \mathrm{~S}_{2}$ 561.1170. Found 561.1175.

2-(4-(4-Methoxybenzoyl)-2,5-diphenyl-1,3-dithiol-2-yl)-1-(4-methoxyphenyl)ethan-1-one (B4) 


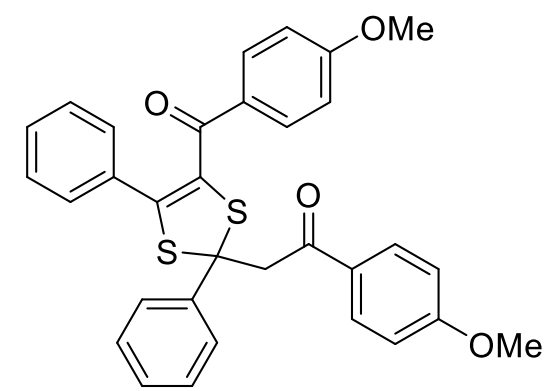

Eluent: hexane/ethyl acetate: 19/1 to 9/1.

Bright yellow solid (61 mg, 45\%).

${ }^{1} \mathrm{H}$ NMR $\left(300 \mathrm{MHz}, \mathrm{CDCl}_{3}\right) \delta 8.0-7.9(\mathrm{~m}, 4 \mathrm{H}), 7.6(\mathrm{~d}, J=9.1 \mathrm{~Hz}, 2 \mathrm{H}), 7.4(\mathrm{dd}, J=8.4,6.8 \mathrm{~Hz}, 2 \mathrm{H})$, $7.3(\mathrm{ddd}, J=7.5,4.8,1.7 \mathrm{~Hz}, 2 \mathrm{H}), 7.1(\mathrm{td}, J=5.9,2.8 \mathrm{~Hz}, 2 \mathrm{H}), 6.9(\mathrm{~d}, J=8.4 \mathrm{~Hz}, 2 \mathrm{H}), 6.7(\mathrm{~d}, J=10.2$ $\mathrm{Hz}, 2 \mathrm{H}), 4.5$ (s, 2H), 3.9 (s, 3H), 3.7 (s, 3H).

${ }^{13} \mathrm{C}\left\{{ }^{1} \mathrm{H}\right\} \operatorname{NMR}\left(75 \mathrm{MHz}, \mathrm{CDCl}_{3}\right) \delta 192.2,188.2,162.9,162.2,141.5,140.7,131.3,130.7,129.4,128.7$, $128.2,128.2,127.3,127.1,126.7,126.6,125.4,125.0,112.8,112.3,112.2,65.9,54.5,54.3,49.6$.

HRMS m/z calculated for $[\mathrm{M}+\mathrm{Na}]^{+} \mathrm{C}_{32} \mathrm{H}_{26} \mathrm{NaO}_{4} \mathrm{~S}_{2}$ 561.1170. Found 561.1175.

2-(4-(4-Methoxybenzoyl)-2,5-bis(4-methoxyphenyl)-1,3-dithiol-2-yl)-1-(4-methoxyphenyl)ethan1-one (B5)<smiles>COc1ccc(C(=O)CC2(c3ccc(OC)cc3)SC(C(=O)c3ccc(OC)cc3)=C(c3ccc(OC)cc3)S2)cc1</smiles>

Eluent: hexane/ethyl acetate: $19 / 1$ to $9 / 1$.

Bright yellow solid (70 mg, 47\%). 
${ }^{1} \mathrm{H}$ NMR $\left(300 \mathrm{MHz}, \mathrm{CDCl}_{3}\right) \delta 7.9(\mathrm{~d}, J=8.9 \mathrm{~Hz}, 2 \mathrm{H}), 7.8(\mathrm{~d}, J=8.9 \mathrm{~Hz}, 2 \mathrm{H}), 7.6(\mathrm{~d}, J=8.8 \mathrm{~Hz}, 2 \mathrm{H})$, $7.2(\mathrm{~d}, J=8.8 \mathrm{~Hz}, 2 \mathrm{H}), 6.9(\mathrm{dd}, J=11.9,8.9 \mathrm{~Hz}, 4 \mathrm{H}), 6.6(\mathrm{t}, J=8.9 \mathrm{~Hz}, 3 \mathrm{H}), 4.4(\mathrm{~s}, 2 \mathrm{H}), 3.9(\mathrm{~s}, 3 \mathrm{H})$, $3.8(\mathrm{~s}, 3 \mathrm{H}), 3.8(\mathrm{~s}, 3 \mathrm{H}), 3.7(\mathrm{~s}, 3 \mathrm{H})$.

${ }^{13} \mathrm{C}\left\{{ }^{1} \mathrm{H}\right\} \mathrm{NMR}\left(75 \mathrm{MHz}, \mathrm{CDCl}_{3}\right) \delta 193.4,189.5,163.9,163.2,160.3,158.9,142.4,134.4,131.7,131.6$, $131.3,131.1,131.0,130.5,130.4,130.3,130.1,129.4,129.3,129.2,129.1,129.0,127.9,124.9,124.6$, $113.9,113.6,113.4,113.3,66.2,55.5,55.4,55.3,55.2,50.4$.

HRMS m/z calculated for $[\mathrm{M}+\mathrm{Na}]^{+} \mathrm{C}_{34} \mathrm{H}_{30} \mathrm{NaO}_{6} \mathrm{~S}_{2}$ 621.1381. Found 621.1392.

\section{2-(4-Benzoyl-2,5-bis(4-fluorophenyl)-1,3-dithiol-2-yl)-1-phenylethan-1-one (B6)}<smiles>O=C(CC1(c2ccc(F)cc2)SC(C(=O)c2ccccc2)=C(c2ccc(F)cc2)S1)c1ccccc1</smiles>

Eluent: hexane/ethyl acetate: $19 / 1$ to $9 / 1$.

Bright yellow solid (75 mg, 58\%).

${ }^{1} \mathrm{H}$ NMR $\left(500 \mathrm{MHz}, \mathrm{CDCl}_{3}\right) \delta 7.9(\mathrm{~d}, J=8.2 \mathrm{~Hz}, 2 \mathrm{H}), 7.9-7.8(\mathrm{~m}, 2 \mathrm{H}), 7.6(\mathrm{t}, J=7.4 \mathrm{~Hz}, 1 \mathrm{H}), 7.5(\mathrm{t}$, $J=7.9 \mathrm{~Hz}, 3 \mathrm{H}), 7.3(\mathrm{t}, J=7.4 \mathrm{~Hz}, 1 \mathrm{H}), 7.2-7.2(\mathrm{~m}, 1 \mathrm{H}), 7.1(\mathrm{t}, J=7.9 \mathrm{~Hz}, 2 \mathrm{H}), 7.1(\mathrm{t}, J=8.5 \mathrm{~Hz}$, 2H), $6.8(\mathrm{t}, J=8.6 \mathrm{~Hz}, 2 \mathrm{H}), 4.5(\mathrm{~s}, 2 \mathrm{H})$.

${ }^{13} \mathrm{C}\left\{{ }^{1} \mathrm{H}\right\}$ NMR $\left(126 \mathrm{MHz}, \mathrm{CDCl}_{3}\right) \delta 194.7,190.2,163.6(\mathrm{~d}, J=132.1 \mathrm{~Hz}), 161.6(\mathrm{~d}, J=129.2 \mathrm{~Hz})$, $143.1,138.2,136.5,136.0,133.9,132.8,131.8,131.7,129.2,128.8,128.5(\mathrm{~d}, J=8.1 \mathrm{~Hz}), 128.1(\mathrm{~d}, J=$ $4.3 \mathrm{~Hz}), 115.3(\mathrm{~d}, J=4.3 \mathrm{~Hz}), 115.2(\mathrm{~d}, J=4.3 \mathrm{~Hz}), 65.9,51.1$.

${ }^{19} \mathrm{~F}$ NMR $\left(471 \mathrm{MHz}, \mathrm{CDCl}_{3}\right) \delta-110.2,-114.2$.

HRMS m/z calculated for $[\mathrm{M}+\mathrm{Na}]^{+} \mathrm{C}_{30} \mathrm{H}_{20} \mathrm{~F}_{2} \mathrm{NaO}_{2} \mathrm{~S}_{2}$ 537.0770. Found 537.0775. 


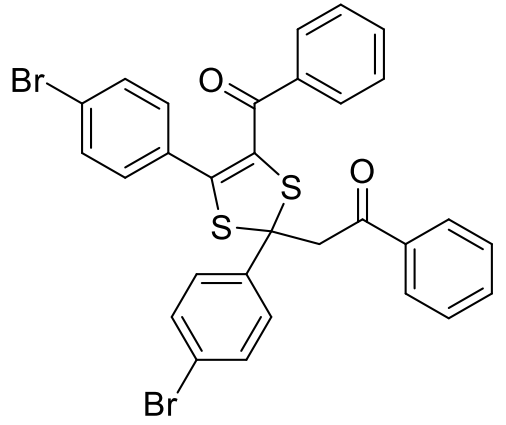

Eluent: hexane/ethyl acetate: $19 / 1$ to $9 / 1$.

Bright yellow solid (80 mg, 50\%).

${ }^{1} \mathrm{H}$ NMR $\left(500 \mathrm{MHz}, \mathrm{CDCl}_{3}\right) \delta 7.92(\mathrm{dd}, J=8.1,1.4 \mathrm{~Hz}, 2 \mathrm{H}), 7.74(\mathrm{~d}, J=8.6 \mathrm{~Hz}, 1 \mathrm{H}), 7.60(\mathrm{t}, J=7.4$ $\mathrm{Hz}, 1 \mathrm{H}), 7.54-7.43(\mathrm{~m}, 5 \mathrm{H}), 7.38-7.31(\mathrm{~m}, 1 \mathrm{H}), 7.25-7.10(\mathrm{~m}, 4 \mathrm{H}), 7.08(\mathrm{~d}, J=8.5 \mathrm{~Hz}, 1 \mathrm{H}), 4.45$ (s, 2H).

${ }^{13} \mathrm{C}\left\{{ }^{1} \mathrm{H}\right\} \mathrm{NMR}\left(126 \mathrm{MHz}, \mathrm{CDCl}_{3}\right) \delta 194.5,190.0,142.3,141.6,136.4,135.9,134.0,132.9,131.5,131.4$, $131.2,130.8,129.2,128.8,128.3,128.2,128.1,127.0,123.9,122.1,66.2,51.0$.

HRMS m/z calculated for $[\mathrm{M}+\mathrm{Na}]^{+} \mathrm{C}_{30} \mathrm{H}_{20} \mathrm{Br}_{2} \mathrm{NaO}_{2} \mathrm{~S}_{2}$ 656.9169. Found 656.9176.

2-(4-(4-Chlorobenzoyl)-2,5-diphenyl-1,3-dithiol-2-yl)-1-(4-chlorophenyl)ethan-1-one (B8)<smiles>O=C(CC1(c2ccccc2)SC(C(=O)c2ccc(Cl)cc2)=C(c2ccccc2)S1)c1ccc(Cl)cc1</smiles>

Eluent: hexane/ethyl acetate: $19 / 1$ to $9 / 1$.

Bright yellow solid (67 mg, 49\%).

${ }^{1} \mathrm{H}$ NMR $\left(500 \mathrm{MHz}, \mathrm{CDCl}_{3}\right) \delta 7.9-7.8(\mathrm{~m}, 5 \mathrm{H}), 7.4-7.4(\mathrm{~m}, 5 \mathrm{H}), 7.4(\mathrm{~d}, J=8.0 \mathrm{~Hz}, 2 \mathrm{H}), 7.3-7.3$ (m, 1H), $7.2-7.2(\mathrm{~m}, 2 \mathrm{H}), 7.2-7.1(\mathrm{~m}, 1 \mathrm{H}), 7.1(\mathrm{~d}, J=8.4 \mathrm{~Hz}, 2 \mathrm{H}), 7.1(\mathrm{~d}, J=9.4 \mathrm{~Hz}, 2 \mathrm{H}), 4.4(\mathrm{~s}$, $2 \mathrm{H})$. 
${ }^{13} \mathrm{C}\left\{{ }^{1} \mathrm{H}\right\} \operatorname{NMR}\left(126 \mathrm{MHz}, \mathrm{CDCl}_{3}\right) \delta 193.6,189.1,145.0,142.1,140.4,138.9,135.1,134.5,131.9,130.6$, $129.9,129.8,129.6,129.1,128.5,128.3,128.1,126.5,126.2,66.5,51.2$

HRMS m/z calculated for $[\mathrm{M}+\mathrm{Na}]^{+} \mathrm{C}_{30} \mathrm{H}_{20} \mathrm{Cl}_{2} \mathrm{NaO}_{2} \mathrm{~S}_{2}$ 569.0179. Found 569.0184.

2-(4-Benzoyl-2,5-bis(4-chlorophenyl)-1,3-dithiol-2-yl)-1-phenylethan-1-one (B9)<smiles>O=C(CC1(c2ccc(Cl)cc2)SC(C(=O)c2ccccc2)=C(c2ccc(Cl)cc2)S1)c1ccccc1</smiles>

Eluent: hexane/ethyl acetate: $19 / 1$ to $9 / 1$.

Bright yellow solid (71 mg, 52\%).

${ }^{1} \mathrm{H}$ NMR $\left(500 \mathrm{MHz}, \mathrm{CDCl}_{3}\right) \delta 7.9(\mathrm{~d}, J=6.3 \mathrm{~Hz}, 2 \mathrm{H}), 7.8(\mathrm{~d}, J=8.7 \mathrm{~Hz}, 2 \mathrm{H}), 7.6(\mathrm{t}, J=7.7 \mathrm{~Hz}, 1 \mathrm{H})$, $7.5(\mathrm{~d}, J=7.1 \mathrm{~Hz}, 2 \mathrm{H}), 7.5(\mathrm{t}, J=7.8 \mathrm{~Hz}, 2 \mathrm{H}), 7.4-7.3(\mathrm{~m}, 3 \mathrm{H}), 7.2-7.1(\mathrm{~m}, 4 \mathrm{H}), 7.1(\mathrm{~d}, J=8.5 \mathrm{~Hz}$, $2 \mathrm{H}), 4.5(\mathrm{~s}, 2 \mathrm{H})$.

${ }^{13} \mathrm{C}\left\{{ }^{1} \mathrm{H}\right\} \mathrm{NMR}\left(126 \mathrm{MHz}, \mathrm{CDCl}_{3}\right) \delta 194.6,190.2,142.5,141.1,136.5,135.9,135.6,134.0,133.9,133.0$, $131.1,130.4,129.8,129.6,129.3,129.1,128.9,128.6,128.6,128.5,128.4,128.3,128.2,128.1,128.0$, 127.0, 66.1, 51.1.

HRMS m/z calculated for $[\mathrm{M}+\mathrm{Na}]^{+} \mathrm{C}_{30} \mathrm{H}_{20} \mathrm{Cl}_{2} \mathrm{NaO}_{2} \mathrm{~S}_{2}$ 569.0179. Found 569.0188.

2-(4-Benzoyl-2,5-bis(3-bromophenyl)-1,3-dithiol-2-yl)-1-phenylethan-1-one (B10) 
<smiles>O=C(CC1(c2cccc(Br)c2)SC(C(=O)c2ccccc2)=C(c2cccc(Br)c2)S1)c1ccccc1</smiles>

Eluent: hexane/ethyl acetate: 19/1 to 9/1.

Bright yellow solid (68 mg, 43\%).

${ }^{1} \mathrm{H}$ NMR $\left(500 \mathrm{MHz}, \mathrm{CDCl}_{3}\right) \delta 7.97(\mathrm{t}, J=2.0 \mathrm{~Hz}, 1 \mathrm{H}), 7.89-7.83(\mathrm{~m}, 2 \mathrm{H}), 7.69(\mathrm{dd}, J=8.0,2.0 \mathrm{~Hz}$, $1 \mathrm{H}), 7.52(\mathrm{t}, J=7.5 \mathrm{~Hz}, 1 \mathrm{H}), 7.41(\mathrm{dd}, J=8.2,6.7 \mathrm{~Hz}, 5 \mathrm{H}), 7.35(\mathrm{dd}, J=8.0,1.8 \mathrm{~Hz}, 1 \mathrm{H}), 7.27(\mathrm{~d}, J=$ $1.9 \mathrm{~Hz}, 1 \mathrm{H}), 7.26-7.21(\mathrm{~m}, 1 \mathrm{H}), 7.20-7.15(\mathrm{~m}, 3 \mathrm{H}), 7.12-7.05(\mathrm{~m}, 3 \mathrm{H}), 6.86(\mathrm{t}, J=7.9 \mathrm{~Hz}, 1 \mathrm{H})$, $4.40(\mathrm{~d}, J=1.5 \mathrm{~Hz}, 2 \mathrm{H})$.

${ }^{13} \mathrm{C}\left\{{ }^{1} \mathrm{H}\right\}$ NMR $\left(126 \mathrm{MHz}, \mathrm{CDCl}_{3}\right) \delta 194.4,190.0,144.8,141.9,136.5,135.8,134.0,133.6,132.9,132.7$, $132.5,131.1,130.0,129.8,129.6,129.1,128.9,128.3,128.2,127.7,125.0,122.7,122.1,65.8,51.1$.

HRMS m/z calculated for $[\mathrm{M}+\mathrm{Na}]^{+} \mathrm{C}_{30} \mathrm{H}_{20} \mathrm{Br}_{2} \mathrm{NaO}_{2} \mathrm{~S}_{2}$ 656.9169. Found 656.9174.

2-(4-Benzoyl-2,5-bis(4-(trifluoromethyl)phenyl)-1,3-dithiol-2-yl)-1-phenylethan-1-one (B11)<smiles>O=C(CC1(c2ccc(C(F)(F)F)cc2)SC(C(=O)c2ccccc2)=C(c2ccc(C(F)(F)F)cc2)S1)c1ccccc1</smiles>

Eluent: hexane/ethyl acetate: 19/1 to 9/1.

Bright yellow solid (87 mg, 57\%). 
${ }^{1} \mathrm{H}$ NMR $\left(300 \mathrm{MHz}, \mathrm{CDCl}_{3}\right) \delta 8.0(\mathrm{~d}, J=8.2 \mathrm{~Hz}, 2 \mathrm{H}), 8.0(\mathrm{~d}, J=7.6 \mathrm{~Hz}, 2 \mathrm{H}), 7.7(\mathrm{~d}, J=8.3 \mathrm{~Hz}, 2 \mathrm{H})$, $7.6(\mathrm{~d}, J=7.9 \mathrm{~Hz}, 1 \mathrm{H}), 7.6-7.5(\mathrm{~m}, 4 \mathrm{H}), 7.4-7.3(\mathrm{~m}, 6 \mathrm{H}), 7.2(\mathrm{t}, J=7.0 \mathrm{~Hz}, 2 \mathrm{H}), 4.6(\mathrm{~s}, 2 \mathrm{H})$.

${ }^{13} \mathrm{C}\left\{{ }^{1} \mathrm{H}\right\} \mathrm{NMR}\left(75 \mathrm{MHz}, \mathrm{CDCl}_{3}\right) \delta 194.4,189.7,146.5,141.3,136.2,135.6,135.3,134.1,133.2,130.0$ 129.1, 128.9, 128.3, 128.1, 127.0, 125.5 (d, $J=3.8 \mathrm{~Hz}), 125.1(\mathrm{~d}, J=4.1 \mathrm{~Hz}), 66.5,51.2$.

HRMS m/z calculated for $[\mathrm{M}+\mathrm{Na}]^{+} \mathrm{C}_{32} \mathrm{H}_{20} \mathrm{~F}_{6} \mathrm{NaO}_{2} \mathrm{~S}_{2}$ 637.0707. Found 637.0712.

2-(4-(1-Naphthoyl)-2,5-diphenyl-1,3-dithiol-2-yl)-1-(naphthalen-1-yl)ethan-1-one (B12)<smiles>O=C(CC1(c2ccccc2)SC(C(=O)c2cccc3ccccc23)=C(c2ccccc2)S1)c1cccc2ccccc12</smiles>

Eluent: hexane/ethyl acetate: $19 / 1$ to $9 / 1$.

Bright yellow solid (75 mg, 52\%).

${ }^{1} \mathrm{H}$ NMR $\left(500 \mathrm{MHz}, \mathrm{CDCl}_{3}\right) \delta 8.38-8.32(\mathrm{~m}, 1 \mathrm{H}), 8.20-8.10(\mathrm{~m}, 1 \mathrm{H}), 8.00(\mathrm{~d}, J=8.2 \mathrm{~Hz}, 1 \mathrm{H}), 7.96$ $(\mathrm{d}, J=7.5 \mathrm{~Hz}, 2 \mathrm{H}), 7.88-7.81(\mathrm{~m}, 2 \mathrm{H}), 7.64(\mathrm{dd}, J=6.2,3.3 \mathrm{~Hz}, 1 \mathrm{H}), 7.58(\mathrm{~d}, J=8.2 \mathrm{~Hz}, 1 \mathrm{H}), 7.54-$ $7.47(\mathrm{~m}, 3 \mathrm{H}), 7.45-7.38(\mathrm{~m}, 4 \mathrm{H}), 7.33(\mathrm{dd}, J=8.8,7.1 \mathrm{~Hz}, 2 \mathrm{H}), 7.03(\mathrm{t}, J=7.7 \mathrm{~Hz}, 1 \mathrm{H}), 6.99$ (d, $J=$ $7.1 \mathrm{~Hz}, 2 \mathrm{H}), 6.86(\mathrm{t}, J=7.5 \mathrm{~Hz}, 1 \mathrm{H}), 4.61(\mathrm{~d}, J=17.4 \mathrm{~Hz}, 1 \mathrm{H}), 4.56(\mathrm{~d}, J=17.3 \mathrm{~Hz}, 1 \mathrm{H})$.

${ }^{13} \mathrm{C}\left\{{ }^{1} \mathrm{H}\right\}$ NMR $\left(126 \mathrm{MHz}, \mathrm{CDCl}_{3}\right) \delta 198.8,190.7,148.8,142.8,135.4,134.9,133.9,133.4,133.2,131.6$, $131.6,130.6,130.0,129.7,129.3,129.1,128.5,128.4,128.3,128.2,128.0,128.0,127.8,127.3,127.2$, $126.7,126.0,125.6,125.3,124.3,124.0,77.3,77.0,76.8,65.5,54.3$.

HRMS m/z calculated for $[\mathrm{M}+\mathrm{Na}]^{+} \mathrm{C}_{38} \mathrm{H}_{26} \mathrm{NaO}_{2} \mathrm{~S}_{2}$ 601.1272. Found 601.1278.

2-(4-(1-Naphthoyl)-2,5-bis(4-fluorophenyl)-1,3-dithiol-2-yl)-1-(naphthalen-1-yl)ethan-1-one (B13) 
<smiles>O=C(CC1(c2ccc(F)cc2)SC(C(=O)c2cccc3ccccc23)=C(c2ccc(F)cc2)S1)c1cccc2ccccc12</smiles>

Eluent: hexane/ethyl acetate: 19/1 to 9/1.

Bright yellow solid (87 mg, 57\%).

${ }^{1} \mathrm{H}$ NMR $\left(500 \mathrm{MHz}, \mathrm{CDCl}_{3}\right) \delta 8.45-8.36(\mathrm{~m}, 1 \mathrm{H}), 8.07(\mathrm{~d}, J=8.1 \mathrm{~Hz}, 1 \mathrm{H}), 8.01(\mathrm{~d}, J=8.2 \mathrm{~Hz}, 1 \mathrm{H})$, $7.97(\mathrm{dd}, J=8.8,5.2 \mathrm{~Hz}, 2 \mathrm{H}), 7.85(\mathrm{dd}, J=8.5,6.0 \mathrm{~Hz}, 2 \mathrm{H}), 7.68(\mathrm{~d}, J=6.8 \mathrm{~Hz}, 1 \mathrm{H}), 7.64(\mathrm{~d}, J=8.2$ $\mathrm{Hz}, 1 \mathrm{H}), 7.53(\mathrm{td}, J=5.5,4.8,3.2 \mathrm{~Hz}, 2 \mathrm{H}), 7.51-7.46(\mathrm{~m}, 1 \mathrm{H}), 7.45-7.35(\mathrm{~m}, 2 \mathrm{H}), 7.31(\mathrm{dd}, J=7.1$, $1.1 \mathrm{~Hz}, 1 \mathrm{H}), 7.10(\mathrm{dt}, J=15.1,8.1 \mathrm{~Hz}, 3 \mathrm{H}), 6.93(\mathrm{dd}, J=8.6,5.4 \mathrm{~Hz}, 2 \mathrm{H}), 6.38(\mathrm{t}, J=8.6 \mathrm{~Hz}, 2 \mathrm{H}), 4.56$ (d, $J=17.4 \mathrm{~Hz}, 1 \mathrm{H}), 4.52(\mathrm{~d}, J=17.4 \mathrm{~Hz}, 1 \mathrm{H})$.

${ }^{13} \mathrm{C}\left\{{ }^{1} \mathrm{H}\right\} \mathrm{NMR}\left(126 \mathrm{MHz}, \mathrm{CDCl}_{3}\right) \delta 198.6,190.5,163.5(\mathrm{~d}, J=76.9 \mathrm{~Hz}), 161.5(\mathrm{~d}, J=74.5 \mathrm{~Hz}), 147.3$, $138.9(\mathrm{~d}, J=3.2 \mathrm{~Hz}), 135.3,134.5,133.7,133.2,131.7,131.1(\mathrm{~d}, J=8.5 \mathrm{~Hz}), 130.5,130.1,130.0,128.7$ (d, $J=8.2 \mathrm{~Hz}), 128.5,128.3,128.2(\mathrm{~d}, J=5.1 \mathrm{~Hz}), 128.0,127.5,127.5,126.8,126.2,125.5,125.0$, 124.3, 124.1, $115.3(\mathrm{~d}, J=21.9 \mathrm{~Hz}), 114.4(\mathrm{~d}, J=22.0 \mathrm{~Hz}), 65.0,54.2$.

${ }^{19} \mathrm{~F}$ NMR $\left(471 \mathrm{MHz}, \mathrm{CDCl}_{3}\right) \delta-110.52,-114.05$.

HRMS m/z calculated for $[\mathrm{M}+\mathrm{Na}]^{+} \mathrm{C}_{38} \mathrm{H}_{24} \mathrm{~F}_{2} \mathrm{NaO}_{2} \mathrm{~S}_{2}$ 637.1083. Found 637.1085.

2-(4-(1-Naphthoyl)-2,5-bis(3-chlorophenyl)-1,3-dithiol-2-yl)-1-(naphthalen-1-yl)ethan-1-one (B14) 
<smiles>O=C(CC1(c2cccc(Cl)c2)SC(C(=O)c2cccc3ccccc23)=C(c2cccc(Cl)c2)S1)c1cccc2ccccc12</smiles>

Eluent: hexane/ethyl acetate: 19/1 to 9/1.

Bright yellow solid (84 mg, 55\%).

${ }^{1} \mathrm{H}$ NMR $\left(500 \mathrm{MHz}, \mathrm{CDCl}_{3}\right) \delta 8.4(\mathrm{~d}, J=8.4 \mathrm{~Hz}, 1 \mathrm{H}), 8.1(\mathrm{~d}, J=9.5 \mathrm{~Hz}, 1 \mathrm{H}), 8.0-8.0(\mathrm{~m}, 2 \mathrm{H}), 7.9-$ $7.8(\mathrm{~m}, 2 \mathrm{H}), 7.8(\mathrm{~d}, J=7.8 \mathrm{~Hz}, 1 \mathrm{H}), 7.7-7.7(\mathrm{~m}, 1 \mathrm{H}), 7.6(\mathrm{~d}, J=8.2 \mathrm{~Hz}, 1 \mathrm{H}), 7.6-7.5(\mathrm{~m}, 4 \mathrm{H}), 7.4$ (qd, $J=7.0,3.6 \mathrm{~Hz}, 2 \mathrm{H}), 7.4-7.3(\mathrm{~m}, 4 \mathrm{H}), 7.1(\mathrm{t}, J=7.6 \mathrm{~Hz}, 1 \mathrm{H}), 6.9(\mathrm{~s}, 1 \mathrm{H}), 6.8(\mathrm{~d}, J=8.1 \mathrm{~Hz}, 2 \mathrm{H})$, $6.6(\mathrm{t}, J=7.9 \mathrm{~Hz}, 1 \mathrm{H}), 4.6(\mathrm{~d}, J=17.6 \mathrm{~Hz}, 1 \mathrm{H}), 4.5(\mathrm{~d}, J=17.6 \mathrm{~Hz}, 1 \mathrm{H})$.

${ }^{13} \mathrm{C}\left\{{ }^{1} \mathrm{H}\right\} \operatorname{NMR}\left(126 \mathrm{MHz}, \mathrm{CDCl}_{3}\right) \delta 198.3,190.3,146.3,145.3,135.2,134.6,134.2,134.0,133.8,133.5$, $133.2,132.8,131.9,130.9,130.4,130.0,129.8,129.2,129.2,128.5,128.4,128.3,128.2,128.1,128.0$, $127.5,127.3,127.2,126.8,126.3,125.5,125.0,124.7,124.3,124.1,64.9,54.0$.

HRMS m/z calculated for $[\mathrm{M}+\mathrm{Na}]^{+} \mathrm{C}_{38} \mathrm{H}_{24} \mathrm{Cl}_{2} \mathrm{NaO}_{2} \mathrm{~S}_{2}$ 669.0492. Found 669.0798.

2-(4-(1-Naphthoyl)-5-(4-bromophenyl)-2-phenyl-1,3-dithiol-2-yl)-1-(naphthalen-1-yl)ethan-1-one (B15)<smiles>O=C(CC1(c2ccccc2)SC(C(=O)c2cccc3ccccc23)=C(c2ccc(Br)cc2)S1)c1cccc2ccccc12</smiles>

Eluent: hexane/ethyl acetate: $19 / 1$ to $9 / 1$. 
Bright yellow solid (86 mg, 47\%).

${ }^{1} \mathrm{H}$ NMR $\left(500 \mathrm{MHz}, \mathrm{CDCl}_{3}\right) \delta 8.4(\mathrm{~d}, J=9.5 \mathrm{~Hz}, 1 \mathrm{H}), 8.0(\mathrm{~d}, J=8.3 \mathrm{~Hz}, 3 \mathrm{H}), 7.9(\mathrm{dd}, J=7.5,4.1 \mathrm{~Hz}$, 6H), $7.7-7.6(\mathrm{~m}, 3 \mathrm{H}), 7.6(\mathrm{~d}, J=8.7 \mathrm{~Hz}, 3 \mathrm{H}), 7.5-7.5(\mathrm{~m}, 4 \mathrm{H}), 7.5-7.4(\mathrm{~m}, 2 \mathrm{H}), 7.4(\mathrm{ddd}, J=8.1$, 6.7, 1.4 Hz, 2H), $7.3-7.3(\mathrm{~m}, 1 \mathrm{H}), 7.1(\mathrm{t}, J=7.7 \mathrm{~Hz}, 2 \mathrm{H}), 6.8(\mathrm{~d}, J=8.5 \mathrm{~Hz}, 3 \mathrm{H}), 6.8(\mathrm{~d}, J=8.5 \mathrm{~Hz}$, $3 \mathrm{H}), 4.6(\mathrm{~d}, J=17.7 \mathrm{~Hz}, 1 \mathrm{H}), 4.5(\mathrm{~d}, J=17.4 \mathrm{~Hz}, 1 \mathrm{H})$.

${ }^{13} \mathrm{C}\left\{{ }^{1} \mathrm{H}\right\} \operatorname{NMR}\left(126 \mathrm{MHz}, \mathrm{CDCl}_{3}\right) \delta 194.5,190.1,141.7,141.4,135.9,135.5,133.6,133.2,132.4,132.2$, $132.0,131.7,131.5,131.5,131.0,130.1,129.9,129.7,129.4,129.0,128.8,128.7,128.5,128.4,127.9$, $127.7,127.1,126.8,126.7,124.3,123.8,123.5,122.2,67.2,50.9$.

HRMS m/z calculated for $[\mathrm{M}+\mathrm{Na}]^{+} \mathrm{C}_{38} \mathrm{H}_{25} \mathrm{BrNaO}_{2} \mathrm{~S}_{2}$ 679.0377. Found 679.0385.

\section{2-(4-(2-Naphthoyl)-2,5-diphenyl-1,3-dithiol-2-yl)-1-(naphthalen-2-yl)ethan-1-one (B16)}

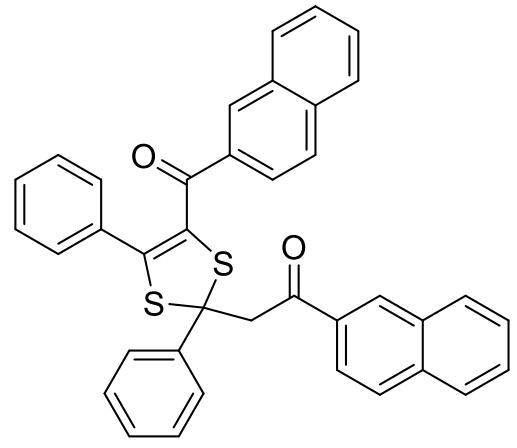

Eluent: hexane/ethyl acetate: 19/1 to 9/1.

Bright yellow solid (77 mg, 53\%).

${ }^{1} \mathrm{H}$ NMR $\left(500 \mathrm{MHz}, \mathrm{CDCl}_{3}\right) \delta 8.49(\mathrm{~d}, J=1.6 \mathrm{~Hz}, 1 \mathrm{H}), 8.02(\mathrm{~d}, J=1.5 \mathrm{~Hz}, 1 \mathrm{H}), 7.99-7.92(\mathrm{~m}, 4 \mathrm{H})$, $7.88(\mathrm{dd}, J=8.5,4.6 \mathrm{~Hz}, 2 \mathrm{H}), 7.71(\mathrm{~d}, J=8.2 \mathrm{~Hz}, 1 \mathrm{H}), 7.68-7.54(\mathrm{~m}, 6 \mathrm{H}), 7.53-7.47(\mathrm{~m}, 1 \mathrm{H}), 7.44$ $-7.38(\mathrm{~m}, 4 \mathrm{H}), 7.34-7.29(\mathrm{~m}, 3 \mathrm{H}), 6.99(\mathrm{dd}, J=5.2,1.9 \mathrm{~Hz}, 3 \mathrm{H}), 4.67$ (s, 2H).

${ }^{13} \mathrm{C}\left\{{ }^{1} \mathrm{H}\right\} \mathrm{NMR}\left(126 \mathrm{MHz}, \mathrm{CDCl}_{3}\right) \delta 194.7,190.5,143.9,142.3,135.8,135.2,133.9,133.6,132.4,132.2$, $132.2,132.0,131.6,130.1,129.7,129.3,129.3,128.9,128.7,128.4,128.3,128.1,128.0,127.8,127.6$, 127.0, 126.6, 126.4, 124.6, 123.6, 67.3, 51.1. 
HRMS m/z calculated for $[\mathrm{M}+\mathrm{Na}]^{+} \mathrm{C}_{38} \mathrm{H}_{26} \mathrm{NaO}_{2} \mathrm{~S}_{2}$ 601.1272. Found 601.1278.

2-(4-(2-Naphthoyl)-2,5-bis(4-fluorophenyl)-1,3-dithiol-2-yl)-1-(naphthalen-2-yl)ethan-1-one (B17)<smiles>O=C(CC1(c2ccc(F)cc2)SC(C(=O)c2ccc3ccccc3c2)=C(c2ccc(F)cc2)S1)c1ccc2ccccc2c1</smiles>

Eluent: hexane/ethyl acetate: $19 / 1$ to $9 / 1$.

Bright yellow solid (77 mg, 50\%).

${ }^{1} \mathrm{H}$ NMR $\left(500 \mathrm{MHz}, \mathrm{CDCl}_{3}\right) \delta 8.48(\mathrm{~d}, J=1.7 \mathrm{~Hz}, 1 \mathrm{H}), 8.01(\mathrm{~s}, 1 \mathrm{H}), 7.96(\mathrm{ddd}, J=13.8,8.3,3.9 \mathrm{~Hz}$, 4H), $7.88(\mathrm{dd}, J=8.3,6.1 \mathrm{~Hz}, 2 \mathrm{H}), 7.74(\mathrm{~d}, J=8.4 \mathrm{~Hz}, 1 \mathrm{H}), 7.69-7.59(\mathrm{~m}, 4 \mathrm{H}), 7.57(\mathrm{~s}, 0 \mathrm{H}), 7.28(\mathrm{dd}$, $J=8.6,5.4 \mathrm{~Hz}, 2 \mathrm{H}), 7.09(\mathrm{t}, J=8.6 \mathrm{~Hz}, 2 \mathrm{H}), 6.70(\mathrm{t}, J=8.6 \mathrm{~Hz}, 2 \mathrm{H}), 4.63(\mathrm{~d}, J=2.7 \mathrm{~Hz}, 2 \mathrm{H})$.

${ }^{13} \mathrm{C}\left\{{ }^{1} \mathrm{H}\right\}$ NMR $\left(126 \mathrm{MHz}, \mathrm{CDCl}_{3}\right) \delta 194.6,190.3,163.6(\mathrm{~d}, J=107.6 \mathrm{~Hz}), 161.6(\mathrm{~d}, J=104.4 \mathrm{~Hz})$, $142.4,138.2,135.9,135.3,133.7,133.4,132.4,132.0,131.6,131.5,131.5,130.1,129.7,129.6,129.0$, $128.8,128.7,128.6,128.6,128.2,127.9,127.7,127.1,126.7,124.5,123.5,115.4(\mathrm{~d}, J=8.5 \mathrm{~Hz}), 115.2$ (d, $J=8.2 \mathrm{~Hz}), 66.8,51.0$.

${ }^{19} \mathrm{~F}$ NMR $\left(471 \mathrm{MHz}, \mathrm{CDCl}_{3}\right) \delta-110.35,-114.11$.

HRMS m/z calculated for $[\mathrm{M}+\mathrm{Na}]^{+} \mathrm{C}_{38} \mathrm{H}_{24} \mathrm{~F}_{2} \mathrm{NaO}_{2} \mathrm{~S}_{2}$ 637.1083. Found 637.1095.

2-(4-(2-Naphthoyl)-2,5-bis(4-bromophenyl)-1,3-dithiol-2-yl)-1-(naphthalen-2-yl)ethan-1-one (B18) 
<smiles>O=C(CC1(c2ccc(Br)cc2)SC(C(=O)c2ccc3ccccc3c2)=C(c2ccc(Br)cc2)S1)c1ccc2ccccc2c1</smiles>

Eluent: hexane/ethyl acetate: 19/1 to 9/1.

Bright yellow solid (79 mg, 43\%).

${ }^{1} \mathrm{H}$ NMR $\left(500 \mathrm{MHz}, \mathrm{CDCl}_{3}\right) \delta 8.47(\mathrm{~d}, J=1.6 \mathrm{~Hz}, 1 \mathrm{H}), 8.00-7.94(\mathrm{~m}, 4 \mathrm{H}), 7.91-7.86(\mathrm{~m}, 2 \mathrm{H}), 7.84$ $(\mathrm{d}, J=8.6 \mathrm{~Hz}, 2 \mathrm{H}), 7.77(\mathrm{~d}, J=8.2 \mathrm{~Hz}, 1 \mathrm{H}), 7.71-7.61(\mathrm{~m}, 4 \mathrm{H}), 7.60-7.51(\mathrm{~m}, 6 \mathrm{H}), 7.48(\mathrm{ddd}, J=$ 8.1, 6.9, $1.2 \mathrm{~Hz}, 1 \mathrm{H}), 7.16(\mathrm{~s}, 4 \mathrm{H}), 4.62(\mathrm{~d}, J=2.2 \mathrm{~Hz}, 2 \mathrm{H})$.

${ }^{13} \mathrm{C}\left\{{ }^{1} \mathrm{H}\right\} \mathrm{NMR}\left(126 \mathrm{MHz}, \mathrm{CDCl}_{3}\right) \delta 194.5,190.1,141.7,141.4,135.9,135.5,133.6,133.2,132.4,132.2$, $132.0,131.7,131.7,131.5,131.5,131.0,130.1,129.9,129.7,129.4,129.0,128.8,128.7,128.5,128.4$, $127.9,127.7,127.1,126.8,126.7,124.3,123.8,123.5,122.2,67.2,50.9$.

HRMS m/z calculated for $[\mathrm{M}+\mathrm{Na}]^{+} \mathrm{C}_{38} \mathrm{H}_{24} \mathrm{Br}_{2} \mathrm{NaO}_{2} \mathrm{~S}_{2}$ 756.9482. Found 756.9488.

2-(4-Benzoyl-2,5-di(naphthalen-2-yl)-1,3-dithiol-2-yl)-1-phenylethan-1-one (B19)<smiles>O=C(CC1(c2ccc3ccccc3c2)SC(C(=O)c2ccccc2)=C(c2ccc3ccccc3c2)S1)c1ccccc1</smiles>

Eluent: hexane/ethyl acetate: $19 / 1$ to $9 / 1$.

Bright yellow solid (69 mg, 48\%). 
${ }^{1} \mathrm{H}$ NMR $\left(500 \mathrm{MHz}, \mathrm{CDCl}_{3}\right) \delta 8.42(\mathrm{~d}, J=2.0 \mathrm{~Hz}, 1 \mathrm{H}), 7.96(\mathrm{dd}, J=8.6,1.9 \mathrm{~Hz}, 3 \mathrm{H}), 7.92-7.85(\mathrm{~m}$, 2H), $7.85-7.79(\mathrm{~m}, 2 \mathrm{H}), 7.71-7.62(\mathrm{~m}, 2 \mathrm{H}), 7.61-7.52(\mathrm{~m}, 3 \mathrm{H}), 7.53-7.38(\mathrm{~m}, 7 \mathrm{H}), 7.29(\mathrm{dd}, J=$ 8.5, 1.8 Hz, 1H), $7.11(\mathrm{t}, J=7.4 \mathrm{~Hz}, 1 \mathrm{H}), 6.98$ (t, $J=7.7 \mathrm{~Hz}, 2 \mathrm{H}), 4.65(\mathrm{~s}, 2 \mathrm{H})$.

${ }^{13} \mathrm{C}\left\{{ }^{1} \mathrm{H}\right\} \mathrm{NMR}\left(126 \mathrm{MHz}, \mathrm{CDCl}_{3}\right) \delta 194.8,190.6,144.2,139.3,136.8,136.2,133.7,133.3,132.8,132.7$, $132.5,132.5,130.0,129.5,129.1,128.8,128.5,128.5,128.2,128.2,127.9,127.7,127.5,127.5,127.0$, $126.8,126.7,126.6,126.5,126.5,126.0,124.2,66.6,51.0$.

HRMS m/z calculated for $[\mathrm{M}+\mathrm{Na}]^{+} \mathrm{C}_{38} \mathrm{H}_{26} \mathrm{NaO}_{2} \mathrm{~S}_{2}$ 601.1272. Found 601.1278.

\section{2-(4-(1-Naphthoyl)-2,5-di(naphthalen-2-yl)-1,3-dithiol-2-yl)-1-(naphthalen-1-yl)ethan-1-one}

(B20)

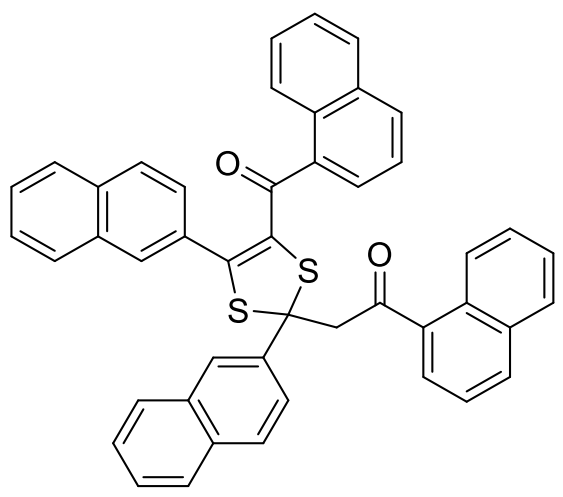

Eluent: hexane/ethyl acetate: $19 / 1$ to $9 / 1$.

Bright yellow solid (78 mg, 46\%).

${ }^{1} \mathrm{H}$ NMR $\left(500 \mathrm{MHz}\right.$, DMSO- $\left.d_{6}\right) \delta 8.6(\mathrm{~d}, J=2.0 \mathrm{~Hz}, 1 \mathrm{H}), 8.4(\mathrm{~d}, J=7.2 \mathrm{~Hz}, 1 \mathrm{H}), 8.2-8.2(\mathrm{~m}, 3 \mathrm{H}), 8.1$ $-8.0(\mathrm{~m}, 3 \mathrm{H}), 8.0(\mathrm{~d}, J=8.1 \mathrm{~Hz}, 1 \mathrm{H}), 8.0-7.9(\mathrm{~m}, 1 \mathrm{H}), 7.7-7.7(\mathrm{~m}, 2 \mathrm{H}), 7.6(\mathrm{ddd}, J=20.0,14.6,7.8$ $\mathrm{Hz}, 5 \mathrm{H}), 7.5(\mathrm{dd}, J=13.0,7.9 \mathrm{~Hz}, 2 \mathrm{H}), 7.4-7.3(\mathrm{~m}, 5 \mathrm{H}), 7.3(\mathrm{dd}, J=11.9,8.0 \mathrm{~Hz}, 2 \mathrm{H}), 7.1(\mathrm{dd}, J=$ 8.6, 1.7 Hz, 1H), 6.9 (t, $J=7.6 \mathrm{~Hz}, 1 \mathrm{H}), 5.2(\mathrm{~d}, J=18.3 \mathrm{~Hz}, 1 \mathrm{H}), 5.0(\mathrm{~d}, J=18.2 \mathrm{~Hz}, 1 \mathrm{H})$.

${ }^{13} \mathrm{C}\left\{{ }^{1} \mathrm{H}\right\}$ NMR (126 MHz, DMSO) $\delta 200.2,190.7,148.5,140.5,135.6,134.6,133.9,133.8,133.1$, $132.8,132.7,132.0,131.6,130.2,129.9,129.8,129.7,129.5,129.0,129.0,128.9,128.8,128.5,128.5$, $128.4,128.1,127.9,127.6,127.6,127.5,127.5,127.2,127.2,127.0,126.7,126.6,126.4,126.0,125.4$, $125.3,125.2,125.0,124.4,66.0,53.4$. 
HRMS m/z calculated for $[\mathrm{M}+\mathrm{Na}]^{+} \mathrm{C}_{46} \mathrm{H}_{30} \mathrm{NaO}_{2} \mathrm{~S}_{2}$ 701.1585. Found 701.1592.

2-(2,4-Di(naphthalen-2-yl)-5-(thiophene-2-carbonyl)-1,3-dithiol-2-yl)-1-(thiophen-2-yl)ethan-1one (B21)

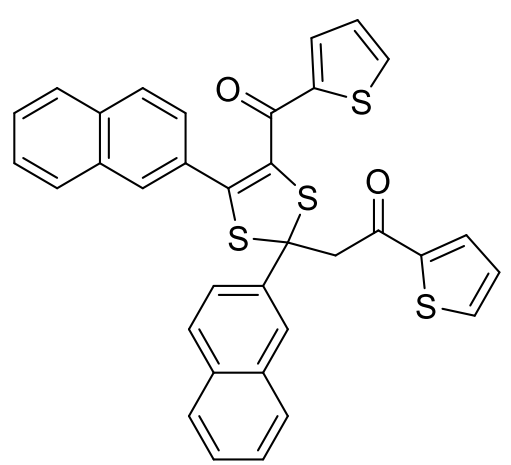

Eluent: hexane/ethyl acetate: 19/1 to 9/1.

Bright yellow solid (62 mg, 42\%).

${ }^{1} \mathrm{H}$ NMR $\left(500 \mathrm{MHz}, \mathrm{CDCl}_{3}\right) \delta 8.4(\mathrm{~d}, J=2.1 \mathrm{~Hz}, 1 \mathrm{H}), 7.9-7.9(\mathrm{~m}, 2 \mathrm{H}), 7.9-7.8(\mathrm{~m}, 2 \mathrm{H}), 7.8-7.7$ $(\mathrm{m}, 1 \mathrm{H}), 7.6(\mathrm{~d}, J=4.9 \mathrm{~Hz}, 1 \mathrm{H}), 7.6(\mathrm{~d}, J=8.5 \mathrm{~Hz}, 1 \mathrm{H}), 7.5-7.4(\mathrm{~m}, 5 \mathrm{H}), 7.4(\mathrm{dd}, J=8.6,1.9 \mathrm{~Hz}, 1 \mathrm{H})$ $7.4(\mathrm{~d}, J=4.9 \mathrm{~Hz}, 1 \mathrm{H}), 7.3(\mathrm{~d}, J=3.8 \mathrm{~Hz}, 1 \mathrm{H}), 7.1(\mathrm{t}, J=4.4 \mathrm{~Hz}, 1 \mathrm{H}), 6.6(\mathrm{t}, J=4.4 \mathrm{~Hz}, 1 \mathrm{H}), 4.5(\mathrm{~d}, J$ $=17.0 \mathrm{~Hz}, 1 \mathrm{H}), 4.5(\mathrm{~d}, J=17.1 \mathrm{~Hz}, 1 \mathrm{H})$.

${ }^{13} \mathrm{C}\left\{{ }^{1} \mathrm{H}\right\}$ NMR $\left(126 \mathrm{MHz}, \mathrm{CDCl}_{3}\right) \delta 187.5,182.4,143.5,142.7,142.2,138.6,134.6,134.5,133.4,132.8$, $132.5,129.7,129.6,128.5,128.5,128.4,128.3,128.0,127.7,127.6,127.5,127.0,126.7,126.6,126.6$, $126.5,126.1,124.2,67.4,51.3$.

HRMS m/z calculated for $[\mathrm{M}+\mathrm{Na}]^{+} \mathrm{C}_{34} \mathrm{H}_{22} \mathrm{NaO}_{2} \mathrm{~S}_{4}$ 613.0400. Found 613.0409.

2-(4-Benzoyl-2,5-di(thiophen-3-yl)-1,3-dithiol-2-yl)-1-phenylethan-1-one (B22)<smiles>O=C(CC1(c2ccsc2)SC(C(=O)c2ccccc2)=C(c2ccsc2)S1)c1ccccc1</smiles> 
Eluent: hexane/ethyl acetate: 19/1 to 9/1.

Bright yellow solid (69 mg, 56\%).

${ }^{1} \mathrm{H}$ NMR $\left(300 \mathrm{MHz}, \mathrm{CDCl}_{3}\right) \delta 8.0-7.9(\mathrm{~m}, 2 \mathrm{H}), 7.7$ - $7.6(\mathrm{~m}, 5 \mathrm{H}), 7.5-7.4(\mathrm{~m}, 2 \mathrm{H}), 7.4-7.4(\mathrm{~m}, 3 \mathrm{H})$, $7.3-7.3(\mathrm{~m}, 2 \mathrm{H}), 7.2(\mathrm{ddt}, J=8.1,6.6,1.2 \mathrm{~Hz}, 3 \mathrm{H}), 7.0(\mathrm{dd}, J=5.0,3.0 \mathrm{~Hz}, 1 \mathrm{H}), 6.9(\mathrm{dd}, J=5.0,1.3$ $\mathrm{Hz}, 1 \mathrm{H}), 4.4(\mathrm{~d}, J=17.5 \mathrm{~Hz}, 1 \mathrm{H}), 4.4(\mathrm{~d}, J=17.5 \mathrm{~Hz}, 1 \mathrm{H})$.

${ }^{13} \mathrm{C}\left\{{ }^{1} \mathrm{H}\right\}$ NMR $\left(75 \mathrm{MHz}, \mathrm{CDCl}_{3}\right) \delta 194.8,190.5,143.7,136.7,136.1,133.8,132.8,132.6,129.3,128.8$, $128.3,128.2,128.0,127.4,126.5,126.3,125.7,123.2,50.3$.

HRMS m/z calculated for $[\mathrm{M}+\mathrm{Na}]^{+} \mathrm{C}_{26} \mathrm{H}_{18} \mathrm{NaO}_{2} \mathrm{~S}_{4}$ 513.0087. Found 513.0093.

2-(2,4-Diphenyl-5-(thiophene-2-carbonyl)-1,3-dithiol-2-yl)-1-(thiophen-2-yl)ethan-1-one (B23)

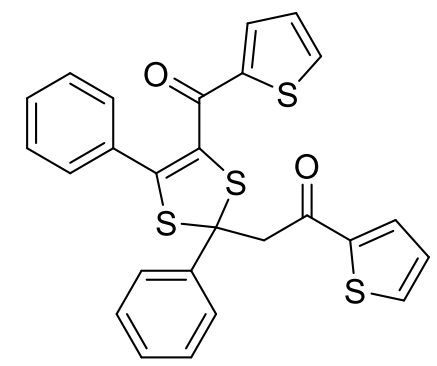

Eluent: hexane/ethyl acetate: $19 / 1$ to $9 / 1$.

Bright yellow solid (72 mg, 59\%).

${ }^{1} \mathrm{H}$ NMR $\left(300 \mathrm{MHz}, \mathrm{CDCl}_{3}\right) \delta 7.9-7.8(\mathrm{~m}, 2 \mathrm{H}), 7.7(\mathrm{dd}, J=3.8,1.2 \mathrm{~Hz}, 1 \mathrm{H}), 7.6(\mathrm{dd}, J=5.0,1.1 \mathrm{~Hz}$, $1 \mathrm{H}), 7.5(\mathrm{dd}, J=4.9,1.2 \mathrm{~Hz}, 1 \mathrm{H}), 7.4(\mathrm{ddd}, J=6.2,4.7,2.7 \mathrm{~Hz}, 4 \mathrm{H}), 7.3-7.3(\mathrm{~m}, 3 \mathrm{H}), 7.2(\mathrm{tq}, J=4.3$, $1.6 \mathrm{~Hz}, 4 \mathrm{H}), 7.1(\mathrm{dd}, J=5.0,3.8 \mathrm{~Hz}, 1 \mathrm{H}), 6.8(\mathrm{dd}, J=4.9,3.8 \mathrm{~Hz}, 1 \mathrm{H}), 4.5(\mathrm{~d}, J=17.1 \mathrm{~Hz}, 1 \mathrm{H}), 4.4(\mathrm{~d}$, $J=17.1 \mathrm{~Hz}, 1 \mathrm{H})$.

${ }^{13} \mathrm{C}\left\{{ }^{1} \mathrm{H}\right\}$ NMR $\left(75 \mathrm{MHz}, \mathrm{CDCl}_{3}\right) \delta 187.6,182.3,143.5,143.4,142.7,142.4,141.6,134.6,134.5,132.6$, $129.7,129.6,128.4,128.4,128.3,128.1,127.7,126.6,125.3,67.4,51.5$.

HRMS m/z calculated for $[\mathrm{M}+\mathrm{Na}]^{+} \mathrm{C}_{26} \mathrm{H}_{18} \mathrm{NaO}_{2} \mathrm{~S}_{4}$ 513.0087. Found 513.0098. 


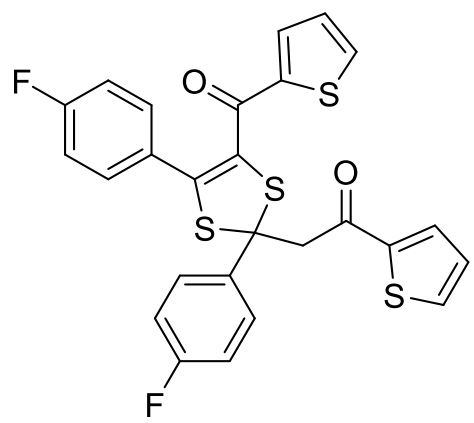

Eluent: hexane/ethyl acetate: 19/1 to 9/1.

Bright yellow solid (60 mg, 46\%).

${ }^{1} \mathrm{H}$ NMR $\left(500 \mathrm{MHz}, \mathrm{CDCl}_{3}\right) \delta 7.8(\mathrm{dd}, J=8.8,5.2 \mathrm{~Hz}, 2 \mathrm{H}), 7.7(\mathrm{~d}, J=3.8 \mathrm{~Hz}, 1 \mathrm{H}), 7.7(\mathrm{~d}, J=5.0 \mathrm{~Hz}$, $1 \mathrm{H}), 7.5(\mathrm{dd}, J=5.0,1.1 \mathrm{~Hz}, 1 \mathrm{H}), 7.3(\mathrm{dd}, J=8.6,5.4 \mathrm{~Hz}, 2 \mathrm{H}), 7.1(\mathrm{t}, J=4.4 \mathrm{~Hz}, 1 \mathrm{H}), 7.0(\mathrm{t}, J=8.6$ Hz, 2H), 6.9 (t, $J=8.6 \mathrm{~Hz}, 2 \mathrm{H}), 6.8(\mathrm{t}, J=4.4 \mathrm{~Hz}, 1 \mathrm{H}), 4.4(\mathrm{~d}, J=17.0 \mathrm{~Hz}, 1 \mathrm{H}), 4.3(\mathrm{~d}, J=17.0 \mathrm{~Hz}$, $1 \mathrm{H})$.

${ }^{13} \mathrm{C}\left\{{ }^{1} \mathrm{H}\right\}$ NMR $\left(126 \mathrm{MHz}, \mathrm{CDCl}_{3}\right) \delta 187.4,182.0,163.7(\mathrm{~d}, J=127.8 \mathrm{~Hz}), 161.7(\mathrm{~d}, J=125.1 \mathrm{~Hz})$, 143.3, 142.5, 141.0, 137.5 (d, $J=3.3 \mathrm{~Hz}), 134.8$ (d, $J=9.8 \mathrm{~Hz}), 134.4,132.6,131.5$ (d, $J=8.5 \mathrm{~Hz})$, $128.5(\mathrm{~d}, J=8.1 \mathrm{~Hz}), 128.3,127.8,125.4,115.6(\mathrm{~d}, J=21.9 \mathrm{~Hz}), 115.3(\mathrm{~d}, J=21.7 \mathrm{~Hz}), 66.9,51.3$.

${ }^{19} \mathrm{~F}$ NMR (471 MHz, $\left.\mathrm{CDCl}_{3}\right) \delta-110.2,-113.9$.

HRMS m/z calculated for $[\mathrm{M}+\mathrm{Na}]^{+} \mathrm{C}_{26} \mathrm{H}_{16} \mathrm{~F}_{2} \mathrm{NaO}_{2} \mathrm{~S}_{4}$ 548.9899. Found 548.9890.

1-(Benzo[b]thiophen-2-yl)-2-(4-(benzo[b]thiophene-2-carbonyl)-2,5-bis(4-fluorophenyl)-1,3dithiol-2-yl)ethan-1-one (B25) 


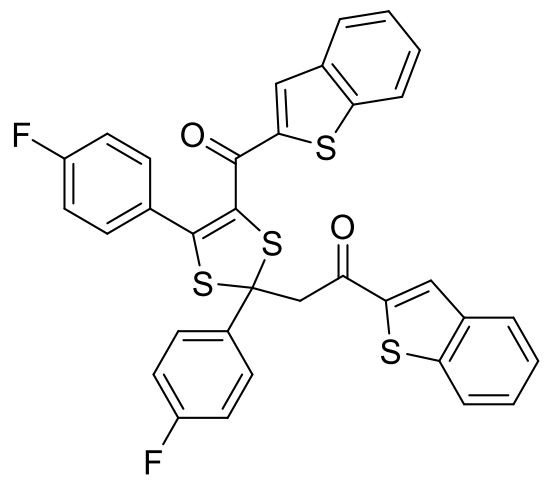

Eluent: hexane/ethyl acetate: $19 / 1$ to $9 / 1$.

Bright yellow solid (61 mg, 39\%).

${ }^{1} \mathrm{H}$ NMR $\left(500 \mathrm{MHz}, \mathrm{CDCl}_{3}\right) \delta 8.0(\mathrm{~s}, 1 \mathrm{H}), 7.9-7.8(\mathrm{~m}, 5 \mathrm{H}), 7.8(\mathrm{~d}, J=8.2 \mathrm{~Hz}, 1 \mathrm{H}), 7.6(\mathrm{~d}, J=8.0 \mathrm{~Hz}$, 1H), $7.5(\mathrm{~s}, 1 \mathrm{H}), 7.5(\mathrm{~d}, J=7.2 \mathrm{~Hz}, 1 \mathrm{H}), 7.4-7.4(\mathrm{~m}, 4 \mathrm{H}), 7.3(\mathrm{~d}, J=7.8 \mathrm{~Hz}, 1 \mathrm{H}), 7.1(\mathrm{t}, J=8.6 \mathrm{~Hz}$, 2H), $6.9(\mathrm{t}, J=8.6 \mathrm{~Hz}, 2 \mathrm{H}), 4.5(\mathrm{~d}, J=17.0 \mathrm{~Hz}, 1 \mathrm{H}), 4.4(\mathrm{~d}, J=16.9 \mathrm{~Hz}, 1 \mathrm{H})$.

${ }^{13} \mathrm{C}\left\{{ }^{1} \mathrm{H}\right\}$ NMR $\left(126 \mathrm{MHz}, \mathrm{CDCl}_{3}\right) \delta 189.0,183.4,164.3,163.3,162.3,161.3,142.8,142.8,142.7,142.0$, $141.6,138.9,138.5,137.2,137.2,131.8,131.5,131.4,130.0,128.7,128.6,128.0,127.7,126.2,126.0$, $125.3,125.1,123.0,122.8,115.8,115.6,115.5,115.3,67.5,51.2$.

${ }^{19} \mathrm{~F}$ NMR $\left(471 \mathrm{MHz}, \mathrm{CDCl}_{3}\right) \delta-109.92,-113.60$.

HRMS m/z calculated for $[\mathrm{M}+\mathrm{Na}]^{+} \mathrm{C}_{34} \mathrm{H}_{20} \mathrm{~F}_{2} \mathrm{NaO}_{2} \mathrm{~S}_{4}$ 649.0212. Found 649.0218.

\section{2-(4-Benzoyl-2,5-di(pyridin-4-yl)-1,3-dithiol-2-yl)-1-phenylethan-1-one (B26)}<smiles>O=C(CC1(c2ccncc2)SC(C(=O)c2ccccc2)=C(c2ccncc2)S1)c1ccccc1</smiles>

Eluent: hexane/ethyl acetate: $19 / 1$ to $9 / 1$.

Bright yellow solid (52 mg, 43\%). 
${ }^{1} \mathrm{H}$ NMR $\left(500 \mathrm{MHz}, \mathrm{CDCl}_{3}\right) \delta 8.65(\mathrm{~d}, J=5.4 \mathrm{~Hz}, 1 \mathrm{H}), 8.35(\mathrm{~d}, J=5.2 \mathrm{~Hz}, 2 \mathrm{H}), 7.90(\mathrm{~d}, J=7.7 \mathrm{~Hz}$, 2H), $7.76(\mathrm{~d}, J=6.8 \mathrm{~Hz}, 2 \mathrm{H}), 7.60(\mathrm{t}, J=7.4 \mathrm{~Hz}, 1 \mathrm{H}), 7.55(\mathrm{~d}, J=7.6 \mathrm{~Hz}, 2 \mathrm{H}), 7.47$ (t, $J=7.7 \mathrm{~Hz}, 2 \mathrm{H})$, 7.38 (t, $J=7.3 \mathrm{~Hz}, 2 \mathrm{H}), 7.20$ (t, $J=7.7 \mathrm{~Hz}, 2 \mathrm{H}), 7.09$ (d, $J=6.8 \mathrm{~Hz}, 2 \mathrm{H}), 4.49$ (s, 2H).

${ }^{13} \mathrm{C}\left\{{ }^{1} \mathrm{H}\right\} \mathrm{NMR}\left(126 \mathrm{MHz}, \mathrm{CDCl}_{3}\right) \delta 194.1,189.5,151.7,150.2,149.9,139.2,138.6,135.9,135.5,134.2$, $133.7,129.3,129.2,128.9,128.5,128.1,123.5,121.1,66.1,50.7$.

HRMS m/z calculated for $[\mathrm{M}+\mathrm{Na}]^{+} \mathrm{C}_{28} \mathrm{H}_{20} \mathrm{~N}_{2} \mathrm{NaO}_{2} \mathrm{~S}_{2}$ 503.0864. Found 503.0870.

2-(4-Benzoyl-2,5-di(pyridin-3-yl)-1,3-dithiol-2-yl)-1-phenylethan-1-one (B27)<smiles>O=C(CC1(c2cccnc2)SC(C(=O)c2ccccc2)=C(c2cccnc2)S1)c1ccccc1</smiles>

Eluent: hexane/ethyl acetate: $19 / 1$ to $9 / 1$.

Bright yellow solid (58 mg, 48\%).

${ }^{1} \mathrm{H}$ NMR $\left(500 \mathrm{MHz}, \mathrm{CDCl}_{3}\right) \delta 9.10(\mathrm{~d}, J=2.7 \mathrm{~Hz}, 1 \mathrm{H}), 8.60-8.50(\mathrm{~m}, 1 \mathrm{H}), 8.45(\mathrm{~d}, J=2.4 \mathrm{~Hz}, 1 \mathrm{H})$, $8.34(\mathrm{dd}, J=4.9,1.8 \mathrm{~Hz}, 1 \mathrm{H}), 8.26(\mathrm{dt}, J=8.3,2.1 \mathrm{~Hz}, 1 \mathrm{H}), 7.92(\mathrm{~d}, J=7.8 \mathrm{~Hz}, 2 \mathrm{H}), 7.59$ (d, $J=7.3$ $\mathrm{Hz}, 1 \mathrm{H}), 7.54-7.45(\mathrm{~m}, 5 \mathrm{H}), 7.37-7.30(\mathrm{~m}, 2 \mathrm{H}), 7.16(\mathrm{t}, J=7.7 \mathrm{~Hz}, 2 \mathrm{H}), 7.01(\mathrm{dd}, J=8.0,4.7 \mathrm{~Hz}$, $1 \mathrm{H}), 4.53(\mathrm{~s}, 2 \mathrm{H})$.

${ }^{13} \mathrm{C}\left\{{ }^{1} \mathrm{H}\right\} \mathrm{NMR}\left(126 \mathrm{MHz}, \mathrm{CDCl}_{3}\right) \delta 194.3,189.5,150.3,149.9,149.1,147.8,140.0,138.5,136.8,136.2$, $135.6,134.5,134.1,133.1,129.7,129.2,128.9,128.4,128.3,128.1,123.0,122.8,64.9,50.9$.

HRMS m/z calculated for $[\mathrm{M}+\mathrm{Na}]^{+} \mathrm{C}_{28} \mathrm{H}_{20} \mathrm{~N}_{2} \mathrm{NaO}_{2} \mathrm{~S}_{2}$ 503.0864. Found 503.0870.

2-(4-Benzoyl-2,5-di(pyridin-2-yl)-1,3-dithiol-2-yl)-1-phenylethan-1-one (B28) 
<smiles>O=C(CC1(c2ccccn2)SC(C(=O)c2ccccc2)=C(c2ccccn2)S1)c1ccccc1</smiles>

Eluent: hexane/ethyl acetate: 19/1 to 9/1.

Bright yellow solid (67 mg, 56\%).

${ }^{1} \mathrm{H}$ NMR $\left(500 \mathrm{MHz}, \mathrm{CDCl}_{3}\right) \delta 8.41(\mathrm{~d}, J=3.0 \mathrm{~Hz}, 0 \mathrm{H}), 8.36(\mathrm{~d}, J=8.0 \mathrm{~Hz}, 1 \mathrm{H}), 8.29(\mathrm{~d}, J=3.8 \mathrm{~Hz}$, 1H), $8.04-7.90(\mathrm{~m}, 2 \mathrm{H}), 7.90-7.71(\mathrm{~m}, 3 \mathrm{H}), 7.57(\mathrm{t}, J=7.6 \mathrm{~Hz}, 1 \mathrm{H}), 7.50-7.35(\mathrm{~m}, 3 \mathrm{H}), 7.21(\mathrm{~d}, J$ $=7.9 \mathrm{~Hz}, 1 \mathrm{H}), 7.15(\mathrm{dd}, J=7.6,4.9 \mathrm{~Hz}, 1 \mathrm{H}), 6.97(\mathrm{dd}, J=7.6,4.7 \mathrm{~Hz}, 1 \mathrm{H}), 4.78(\mathrm{dd}, J=17.2,3.5 \mathrm{~Hz}$, $2 \mathrm{H})$.

${ }^{13} \mathrm{C}\left\{{ }^{1} \mathrm{H}\right\}$ NMR $\left(126 \mathrm{MHz}, \mathrm{CDCl}_{3}\right) \delta 195.8,190.2,161.1,150.4,149.0,148.6,137.2,136.9,136.8,136.2$, $136.1,133.5,132.9,129.6,129.2,128.6,128.3,128.2,123.3,122.7,122.3,67.5,51.3$.

HRMS m/z calculated for $[\mathrm{M}+\mathrm{Na}]^{+} \mathrm{C}_{28} \mathrm{H}_{20} \mathrm{~N}_{2} \mathrm{NaO}_{2} \mathrm{~S}_{2}$ 503.0864. Found 503.0870.

2-(2,4-Bis(4-fluorophenyl)-5-nicotinoyl-1,3-dithiol-2-yl)-1-(pyridin-3-yl)ethan-1-one (B29)<smiles>O=C(CC1(c2ccc(F)cc2)SC(C(=O)c2cccnc2)=C(c2ccc(F)cc2)S1)c1cccnc1</smiles>

Eluent: hexane/ethyl acetate: 19/1 to 9/1.

Bright yellow solid (62 mg, 46\%). 
${ }^{1} \mathrm{H}$ NMR $\left(500 \mathrm{MHz}, \mathrm{CDCl}_{3}\right) \delta 9.1(\mathrm{~s}, 1 \mathrm{H}), 8.8(\mathrm{~d}, J=5.3 \mathrm{~Hz}, 1 \mathrm{H}), 8.6(\mathrm{~s}, 1 \mathrm{H}), 8.5(\mathrm{~d}, J=4.9 \mathrm{~Hz}, 1 \mathrm{H})$, $8.2(\mathrm{~d}, J=8.1 \mathrm{~Hz}, 1 \mathrm{H}), 7.8(\mathrm{dd}, J=9.0,5.0 \mathrm{~Hz}, 2 \mathrm{H}), 7.7(\mathrm{~d}, J=7.6 \mathrm{~Hz}, 1 \mathrm{H}), 7.4(\mathrm{dd}, J=8.0,4.5 \mathrm{~Hz}$, 1H), $7.2(\mathrm{dd}, J=8.7,5.2 \mathrm{~Hz}, 2 \mathrm{H}), 7.1(\mathrm{~m}, 3 \mathrm{H}), 6.8(\mathrm{t}, J=8.5 \mathrm{~Hz}, 2 \mathrm{H}), 4.5(\mathrm{~s}, 2 \mathrm{H})$.

${ }^{13} \mathrm{C}\left\{{ }^{1} \mathrm{H}\right\} \mathrm{NMR}\left(126 \mathrm{MHz}, \mathrm{CDCl}_{3}\right) \delta 193.6,188.2,163.8(\mathrm{~d}, J=149.7 \mathrm{~Hz}), 161.8(\mathrm{~d}, J=146.4 \mathrm{~Hz})$, 145.6, $137.7(\mathrm{~d}, J=2.9 \mathrm{~Hz}), 136.0,135.5,132.3,132.1(\mathrm{~d}, J=8.6 \mathrm{~Hz}), 131.3,128.4(\mathrm{~d}, J=8.6 \mathrm{~Hz})$, $127.4(\mathrm{~d}, J=3.3 \mathrm{~Hz}), 126.7,123.8,122.9,115.7$ (d, $J=21.9 \mathrm{~Hz}), 65.4,51.5$.

${ }^{19} \mathrm{~F}$ NMR $\left(471 \mathrm{MHz}, \mathrm{CDCl}_{3}\right) \delta-108.8,-113.4$.

HRMS m/z calculated for $[\mathrm{M}+\mathrm{Na}]^{+} \mathrm{C}_{28} \mathrm{H}_{18} \mathrm{~F}_{2} \mathrm{~N}_{2} \mathrm{NaO}_{2} \mathrm{~S}_{2}$ 539.0675. Found 539.0682.

2-(2,4-Bis(4-bromophenyl)-5-(furan-2-carbonyl)-1,3-dithiol-2-yl)-1-(furan-2-yl)ethan-1-one (B30)<smiles>O=C(CC1(c2ccc(Br)cc2)SC(C(=O)c2ccco2)=C(c2ccc(Br)cc2)S1)c1ccco1</smiles>

Eluent: hexane/ethyl acetate: $19 / 1$ to $9 / 1$.

Bright yellow solid (62 mg, 40\%).

${ }^{1} \mathrm{H} \operatorname{NMR}\left(500 \mathrm{MHz}, \mathrm{CDCl}_{3}\right) \delta 7.7(\mathrm{~d}, J=8.9 \mathrm{~Hz}, 1 \mathrm{H}), 7.6(\mathrm{~d}, J=2.0 \mathrm{~Hz}, 1 \mathrm{H}), 7.5(\mathrm{~d}, J=8.9 \mathrm{~Hz}, 1 \mathrm{H})$, $7.4(\mathrm{~s}, 0 \mathrm{H}), 7.4-7.3(\mathrm{~m}, 2 \mathrm{H}), 7.2-7.2(\mathrm{~m}, 2 \mathrm{H}), 6.9(\mathrm{~d}, J=3.7 \mathrm{~Hz}, 1 \mathrm{H}), 6.5(\mathrm{dd}, J=3.6,1.8 \mathrm{~Hz}, 1 \mathrm{H})$, $6.3(\mathrm{dd}, J=3.6,1.8 \mathrm{~Hz}, 1 \mathrm{H}), 4.3(\mathrm{~d}, J=1.7 \mathrm{~Hz}, 1 \mathrm{H})$.

${ }^{13} \mathrm{C}\left\{{ }^{1} \mathrm{H}\right\} \mathrm{NMR}\left(126 \mathrm{MHz}, \mathrm{CDCl}_{3}\right) \delta 183.5,176.3,152.1,150.9,146.9,146.8,142.7,140.8,131.9,131.6$, $131.6,131.6,131.5,131.5,131.5,131.4,131.3,130.9,130.8,130.8,130.1,129.9,128.3,128.3,128.3$, $125.5,123.9,122.2,120.0,120.0,120.0,118.0,112.7,112.4,66.6,50.3$.

HRMS m/z calculated for $[\mathrm{M}+\mathrm{Na}]^{+} \mathrm{C}_{26} \mathrm{H}_{16} \mathrm{Br}_{2} \mathrm{NaO}_{4} \mathrm{~S}_{2}$ 636.8754. Found 636.8760 . 


\section{ASSOCIATED CONTENT}

\section{Supporting information}

The Supporting Information is available free of charge on the

ACS Publications website at DOI: 10.1021/acs.joc.xxxxxxx.

Copies of ${ }^{1} \mathrm{H}$ and ${ }^{13} \mathrm{C}$ NMR spectra data for all new compounds. X-ray crystallographic data for $\mathbf{B 0}$ and B11 (CCDC 1952724 and 1952725, respectively).

This material is available free of charge via the Internet at http://pubs.acs.org.

\section{REFERENCES}

(1) For reviews on the use of elemental sulfur in organic synthesis, see: (a) Nguyen, T. B. Recent Advances in Organic Reactions Involving Elemental Sulfur. Adv. Synth. Catal. 2017, 359, 1106-1130. (b) Nguyen, T. B. Elemental sulfur and molecular iodine as efficient tools for carbon-nitrogen bond formation via redox reactions. Asian J. Org. Chem. 2017, 6, 477.

(2) (a) Iyoda, M.; Hasegawa, M.; Miyake, Y. Bi-TTF, Bis-TTF, and Related TTF Oligomers. Chem. Rev. 2004, 104, 5085-5114. (b) Canavet, D.; Sallé, M.; Zhang, G.; Zhang, D.; Zhu, D. Tetrathiafulvalene (TTF) derivatives: key building-blocks for switchable processes. Chem. Commun. 2009, 2245-2269.

(3) (a) Fleck, N.; Hett, T.; Brode, J.; Meyer, A.; Richert, S.; Schiemann, O. C-C Cross-Coupling Reactions of Trityl Radicals: Spin Density Delocalization, Exchange Coupling, and a Spin Label. J. Org. Chem. 2019, 84, 3293. (b) Liu, Y.; Villamena, F. A.; Rockenbauer, A.; Song, Y. Zweier, J. L. Structural Factors Controlling the Spin-Spin Exchange Coupling: EPR Spectroscopic Studies of Highly Asymmetric Trityl-Nitroxide Biradicals. J. Am. Chem. Soc. 2013, 135, 2350-2356. (c) Hintz, H.; Vanas, A.; Klose, D.; Jeschke, G.; Godt, A. Trityl Radicals with a Combination of the Orthogonal Functional Groups Ethyne and Carboxyl: Synthesis without a Statistical Step and EPR Characterization. J. Org. Chem. 2019, 84, 3304-3320.

(4) Uchimasu, H.; Matsumura, K.; Tsuda, M. ; Kumagai, K.; Akakabe, M.; Fujita, M. J.; Sakai, R. Mellpaladines and dopargimine, novel neuroactive guanidine alkaloids from a Palauan Didemnidae tunicate. Tetrahedron 2016, 72, 7185-7193. 
(5) Rivero, R. A.; Greenlee, W. J.; Chang, R. S. L. Substituted 1,3-benzodioxole \& 1,3-benzodithiole -2- carboxylates and their tetrazole analogs with potent binding affinity to the angiotensin II AT1 receptor. Bioorg. Med. Chem. Lett. 1994, 4, 747-750.

(6) (a) McKinnon, D. M.; Buchshriber, J. M. The Reaction of 5-Unsubstituted-1,2-Dithiole-3-thiones with some Activated Acetylenes. Can. J. Chem. 1971, 49, 3299-3304. (b) Meijer, J.; Vermeer, P.; Bos, H. J. T.; Brandsma, L. Thermal rearrangement of 2-alkynyl alkanedithioates. Recl. Trav. Chim. PaysBas 1973, 92, 1067-1072. (b) Konstantinova, L. S.; Lysov, K. A.; Amelichev, S. A.; Obruchnikova, N. V.; Rakitin, O. A. A one-pot synthesis and 1,3-dipolar cycloaddition of [1,2]dithiolo[4,3-b]indole-3(4H)thiones. Tetrahedron 2009, 65, 2178-2183. (c) Benzyne with dithioesters: Kentaro, O; Akiko, N.; Yoshinobu, Y. Reaction of Dithiolactones with Benzyne: A Novel Synthesis of Benzo-1,3-dithioles. Heterocycles 2008, 75, 1417-1424.

(7) (a) Kim, K.-T.; Okazaki, R.; Inamoto, N. Synthesis and Properties of $o$-Thioquinone Methides Having Ketene Aminal, Ketene Acetal, Ketene Monothioacetal, or Ketene Dithioacetal Group. Bull. Chem. Soc. Jpn. 1979, 52, 3640-3646. (b) Burton D. J.; Inouye Y. Synthesis of Perfluorinated 1,3Dithioles from Fluoroolefins. Chem. Lett. 1982, 11, 201-204. (c) Ngounda, M.; Bozec, H. L.; Dixneuf, P. New Synthesis of 1,3-Dithiole and 1,3-Thiazole-2-thiones Promoted by Iron Complexes. J. Org. Chem. 1982, 47, 4000-4002. (d) Kreitsberga, Y. N.; Vilyuma, é. V.; Khodorkovskii, V. Y.; Neiland, O. Y. Synthesis and transformations of 1,3-dithiol-2-ylideneisopropylidene malonates. Chem. Heterocycl. Compd. 1988, 24, 1339-1342. (e) Hartke, K.; Rettberg, N.; Dutta, D.; Gerber, H. Dithio- und Thionester, 59. Zur Reaktion von aliphatischen Dithiosäure-dianionen mit Schwefelkohlenstoff. Liebigs Ann. Chem. 1993, 1081-1089. (f) Frère, P.; Gorgues, A.; Jubault, M.; Riou, A. ; Gouriou, Y.; Roncali, J. Electrochemically induced intramolecular cyclization of 1,2-bis(1,4-dithiafulven-6-yl)benzenes. Tetrahedron Lett. 1994, 35, 1991-1994. (g) Reimann-Andersen, S,; Pritzkow, H. Sundermeyer. H. Halogen-Kohlenstoff-Schwefel-Verbindungen: Zur Chemie von 1,1,1,4,4,4-Hexafluor-2-buten-2,3bissulfenylchlorid. Chem. Ber. 1994, 127, 533-539. (h) Soliman, A. M.; Sultan, A. A.; EI-Shafei, A. K. 
Synthesis of Some New Dispiro[dipyrano-(2,4'.6,4")bidithiolo(4,f-b.4,5-e)- 4,8-benzoquinones. Monat. Chem. 1995, 126, 615-619.

(8) (a) Mayer, R.; Gebhardt, B. Schwefel-Heterocyclen und Vorstufen, XXXIII. Präparative Synthese und Folgereaktionen des Isotrithions (1.3-Dithiol-thions-(2)) und Isodithions (1.3-Dithiol-ons-(2)). Chem. Ber. 1964, 97, 1298 - 1307. (b) Bock, H.; Rittmeyer, P. Zur Darstellung von BenzodithietDerivaten durch Thermolyse 1,2-Dithio-Substituierter Benzole [Phosphorus Sulfur Silicon Relat. Elem. 1988, 35, 291-308. (c) Ando, W.; Tokitoh, N.; Kabe, Y. Synthesis and Reaction of Novel Sulfur Containing Heterocyclic Systems. Phosphorus Sulfur Silicon Relat. Elem. 1991, 58, 179-205. (d) Gusarova, N. K.; Chernysheva, N. A.; Yas'ko, S. V.; Korchevin, N. A.; Dolgushin, G. V.; Trofimov, B. A. Effect of microwave irradiation on the reaction of elemental sulfur with Phenylacetylene. Doklady Chem. 2004, 399, 240-241. (e) Petrov, V. A.; Marshall, W. Remarkable effect of metal fluoride catalyst on reaction of hexafluoropropene, sulfur and vinyl ethers. Convenient synthesis of 2,2bis(trifluoromethyl)-4-R-thietanes, 3,3-bis(trifluoromethyl)-5-R-1,2- dithiolanes and 2,2bis(trifluoromethyl)-4-R-1,3-dithiolanes. J. Fluorine Chem. 2010, 131, 1144-1155. (f) Arisawa, Mieko; Ichikawa, Takuya; Tanii, Saori; Yamaguchi, M. Synthesis of Symmetrical and Unsymmetrical 1,4Dithiins by Rhodium-Catalyzed Sulfur Addition Reaction to Alkynes. Synthesis 2016, 48, 3107-3119. (g) Petrov, Viacheslav; Dooley, Rebecca J.; Marchione, Alexander A.; Diaz, Elizabeth L.; Clem, Brittany S. Simple protocol for preparation of Diels-Alder adducts of perfluorinated thioketone. $J$. Fluorine Chem. 2019, 225, 1-10.

(9) Thioamides from amines: (a) Nguyen, T. B.; Ermolenko, L.; Almourabit, A. Efficient and Selective Multicomponent Oxidative Coupling of Two Different Aliphatic Primary Amines into Thioamides by Elemental Sulfur. Org. Lett. 2012, 14, 4274-4277. Thiophenes: (b) from acetophenones or cyclopentanone: Nguyen, T. B.; Retailleau, P. Sulfurative Self-Condensation of Ketones and Elemental Sulfur: a Three-Component Access to Thiophenes Catalyzed by Aniline Acid-Base Conjugate Pairs. Green Chem. 2018, 20, 387-390. From acetylenes: (c) Nakayama, J.; Yomoda, R.; Hoshino, M. Reactions of Elemental Sulfur and Selenium with Some Asetylenic Compounds. Formation of 
Thiophenes and Selemophenes. Heterocycles 1987, 26, 2215-2222. (d) Blum, J.; Badrieh, Y.; Shaaya, O.; Meltser, L.; Schumann, H. On the Various Modes of Interaction of Sulfur with Phenylated Diynes. Phosphorus Sulfur Silicon Relat. Elem. 1993, 79, 87. (e) Abdel-Wahab, A. A.; Abdel-Rahman, A. E. Molecular Rearrangements: Part I-Pyrolysis of Dibenzyl Sulphide. Indian J. Chem. B 1981, 20, 636639. (f) Liu, W.; Chen, C.; Liu, H. Synthesis of Polysubstituted Thiophenes via Base-Induced [2+2+1] Cycloaddition Reaction of Alkynes and Elemental Sulfur. Adv. Synth. Catal. 2015, 357, 4050-4054. (g) Alizadeh, A.; Hosseinpour, R. An efficient synthesis of tetraalkyl 2,3,4,5-thiophenetetracarboxylate derivatives. Synthesis, 2009, 1960-1962. (h) From chalcone: Joshi, S.; Dhar, D. N. Indian J. Chem.B, 1987, 26, 179. $o, o^{\prime}$-Diaminodiphenyltrisulfide from $o$-aminothiophenol: (i) Nguyen, T. B.; Ermolenko, L.; Dean, W. A.; Almourabit, A. Benzazoles from Aliphatic Amines and oAmino/Mercaptan/Hydroxyanilines: Elemental Sulfur as a Highly Efficient and Traceless Oxidizing Agent. Org. Lett. 2012, 14, 5948-5951.

(10) (a) Oxidative trimerization of arylacetonitriles: Nguyen, T. B.; Retailleau, P. Sulfur-Promoted DABCO-Catalyzed Oxidative Trimerization of Phenylacetonitriles. J. Org. Chem. 2019, 84, 5907-5912.

(b) Desulfurative trimerization of dibenzyl disulfide: see reference 12.

(11) Tetraphenylthiophenes from: benzyl halides: (a) Przewoski, K.; Voronkov, M. G.; Przewoska, L.; Warnke, Z.; Szafranek, J. Reaction of sulfur with benzyl halides. Chem Sci, Bull. Acad. Sci. USSR div. Chem. Sci. 1989, 38, 579. (b) Lai, Chung-Tin; Hong, J. Aggregation-induced emission in tetraphenylthiophene-derived organic molecules and vinyl polymer. J. Phys. Chem. B 2010, 114, 1030210310. (c) Lai, C.; Chien, R.; Liu, C.; Hong, J. Thermal and spectral stability of fluorescent copolymers containing tetraphenylthiophene-quinoline unit. J. Polym. Sci. A 2011, 49, 2059-2069.

(12) Nguyen, T. B.; Retailleau, P. Sulfur-Promoted Decarboxylative Sulfurative Hexamerization of Phenylacetic Acids: Direct Approach to Hexabenzylidyne Tetrasulfides. Org. Lett. 2019, 21, 279-282.

(13) When no sulfur was incorporated to the final molecules, the reactions could be classified as oxidative self-condensation. 
(14) For selected reactions involving elemental sulfur that could be performed at rt, see: (a) Nguyen, T. B.; Retailleau, P. Cooperative Activating Effect of Tertiary Amine-DMSO on Elemental Sulfur: Direct Access to Thioaurones from 2'-Nitrochalcones under Mild Conditions. Org. Lett. 2018, 20, 186-189. (b) Nguyen, T. B.; Ermolenko, L.; Almourabit, A. Three-Component Reaction between Isocyanides, Aliphatic Amines and Elemental Sulfur: Preparation of Thioureas under Mild Conditions with Complete- Atom Economy. Synthesis 2014, 46, 3172-3179.

(15) (a) Nguyen, T. B.; Nguyen, L. A.; Retailleau P. Strategy for Contiguous Tetramination of Cyclohexanones with o-Phenylenediamines with Elemental Sulfur and DMSO. Org. Lett. 2019, 21, 6570-6574. (b) Nguyen, T. B.; Retailleau P. Sulfur-Promoted Synthesis of 2-Aroylquinazolin-4(3H)ones by Oxidative Condensation of Anthranilamide and Acetophenones. Adv. Synth. Catal. 2019, 361, 3337-3341. (c) Nguyen, T. B.; Retailleau P. Sulfur-Catalyzed Stereo and Regioselective Synthesis of Heteropropellanes via Oxidative Condensation of Cyclohexanones with 2-Aminophenols. Adv. Synth. Catal. 2019, 361, 3588-3592. (d) Nguyen, L. A.; Retailleau P.; Nguyen, T. B. Elemental Sulfur/DMSOPromoted Multicomponent One-pot Synthesis of Malonic Acid Derivatives from Maleic Anhydride and Amines. Adv. Synth. Catal. 2019, 361, 2864-2869. (e) Nguyen, T. B.; Retailleau, P. Sulfur-Promoted Aminative Aromatization of 1,2,3,4-Tetrahydrophenazines with Amines: Flexible Access to 1Aminophenazines. Adv. Synth. Catal. 2018, 360, 2389-2393.

(16) Nguyen, T. T. T.; Le, V. A.; Retailleau P.; Nguyen, T. B. Access to 2-Amino-3-Arylthiophenes by Base-Catalyzed Redox Condensation Reaction Between Arylacetonitriles, Chalcones, and Elemental Sulfur. Adv. Synth. Catal. 2020, 362, 160-165.

(17) (a) Nguyen, T. B.; Retailleau, P. DIPEA-Promoted Reaction of 2-Nitrochalcones with Elemental Sulfur: An Unusual Approach to 2-Benzoylbenzothiophenes. Org. Lett. 2017, 19, 4858-4860. (b) Nguyen, T. B.; Retailleau, P. Redox-Neutral Access to Sultams from 2-Nitrochalcones and Sulfur with Complete Atom Economy. Org. Lett. 2017, 19, 3879-3882.

(18) (a) Timokhina, L. V.; Sokol'nikova, O. V.; Kanitskaya, L. V.; Voronkov, M. G. 5',5',6,6Tetramethyl-6,7-dihydro-3'H-spiro[1,3-benzodithiole-2,1'-cyclohexane]-3',4(5H)-dione. Russian $\quad J$. 
Org. Chem. 2009, 45, 466-467. (b) Dalgaard, L.; Lawesson, S. O. Enethiols. VIII. 3-Mercapto-5,5dimethyl-2-cyclohexen-1-one ("Thiodimedone") and Derivatives. Thermal and Photochemical Rearrangements.. Acta Chem. Scand. B 1974, 28, 1077-1090. 